\title{
M-GWAS for the gut microbiome in Chinese adults illuminates on complex diseases
}

Xiaomin Liu ${ }^{1,2}$, Shanmei Tang ${ }^{1,2}$, Huanzi Zhong ${ }^{1,3}$, Xin Tong ${ }^{1,4}$, Zhuye Jie ${ }^{1,5}$, Qiuxia Ding ${ }^{1}$, Dan Wang ${ }^{1,2}$, Ruidong Guo ${ }^{1}$, Liang Xiao ${ }^{1,6,7}$, Xun $\mathrm{Xu}^{1,2}$, Huanming Yang ${ }^{1,8}$, Jian Wang ${ }^{1,8}$,

Yang Zong ${ }^{1}$, Xiao Liu ${ }^{1}$, Yong Zhang ${ }^{1}$, Susanne Brix ${ }^{1,9}$, Karsten Kristiansen ${ }^{1,3}$, Yong Hou ${ }^{1, \dagger}$, Huijue Jia ${ }^{1,5, \dagger}$, Tao Zhang ${ }^{1,2,3, \dagger}$

1. BGI-Shenzhen, Shenzhen 518083, China;

2. China National Genebank, BGI-Shenzhen, Shenzhen 518120, China;

3. Department of Biology, Ole Maaløes Vej 5, University of Copenhagen, DK-2200 Copenhagen, Denmark

4. Shenzhen Key Laboratory of Cognition and Gene Research, BGI-Shenzhen, Shenzhen 518083, China;

5. Shenzhen Key Laboratory of Human Commensal Microorganisms and Health Research, BGI-Shenzhen, Shenzhen 518083, China;

6. Shenzhen Engineering Laboratory of Detection and Intervention of Human Intestinal Microbiome, BGI-Shenzhen, Shenzhen 518083, China;

7. BGI-Qingdao, BGI-Shenzhen, Qingdao, 266555, China;

8. James D. Watson Institute of Genome Sciences, Hangzhou 310058, China;

9. Center for Biological Sequence Analysis, Department of Systems Biology, Technical University of Denmark, Denmark;

$\dagger$ To whom correspondence should be addressed: T.Z., tao.zhang@genomics.cn; H.J., jiahuijue@genomics.cn or Y.H., houyong@genomics.cn. 


\section{Summary}

The gut microbiome has been established as a key environmental factor to health. Genetic influences on the gut microbiome have been reported, yet, doubts remain as to the significance of genetic associations. Here, we provide shotgun data for whole genome and whole metagenome from a Chinese cohort, identifying no less than $20 \%$ genetic contribution to the gut microbiota. Using common variants-, rare variants- and copy number variations (CNVs)-based association analyses, we identified abundant signals associated with the gut microbiome especially in metabolic, neurological and immunological functions. The controversial concept of enterotypes may have a genetic attribute, with the top 2 loci explaining $11 \%$ of the Prevotella-Bacteroides variances. Stratification according to gender led to the identification of differential associations in males and females. Genetically encoded responses to ectopic presence of oral bacteria in the gut appear to be a common theme in a number of diseases investigated by MWAS (Metagenome-wide association studies). Our two-stage M-GWAS (Microbiome genome-wide association studies) on a total of 1295 individuals unequivocally illustrates that neither microbiome nor GWAS studies could overlook one another in our quest for a better understanding of human health and diseases.

Keywords: whole genome, metagenome, gut microbiome, M-GWAS, gender stratification

\section{Highlights}

M-GWAS using high-depth whole genome identifies contributions from rare variants and CNVs.

Gut microbial modules such as butyrate, amino acids, mucin degradation show genetic associations.

Gender differential M-GWAS underscores differences in metabolic and psychological predispositions.

Some of the MWAS markers for colorectal cancer and cardiometabolic diseases show genetic associations. 


\section{Introduction}

The gut microbiome is increasingly recognized as playing important roles in host health and diseases, exerting influences well beyond the gut (Blacher et al., 2019; Wang and Jia, 2016). However, due to modulations by diet and medication, the gut microbiome is commonly viewed as highly dynamic, whereas disease markers are expected to be stable. Studies in mice (Org et al., 2015) and in human twins (Goodrich et al., 2016; Xie et al., 2016) have observed substantial heritability for some bacteria. Several genome-wide association analysis studies (Blekhman et al., 2015; Bonder et al., 2016; Rothschild et al., 2018; Turpin et al., 2016; Wang et al., 2016) for the gut microbiota (M-GWAS) have found associations between host single nucleotide polymorphisms (SNPs) and individual bacterial taxa, beta diversity or pathways. Yet, doubts remain as to the significance of genetic associations. For example, a recent study (Rothschild et al., 2018) including a heterogeneous population of $\sim 800$ individuals reported that the average heritability of gut microbiome taxa is only $1.9 \%$, in contrast to Wang et al. which identified 42 SNPs that together explained $10 \%$ of the variance of the $\beta$-diversity (Wang et al., 2016). In addition, these studies all used genotyping arrays data for host genome and they all used 16S rRNA gene amplicon sequencing except for one study which had low-depth shotgun data for fecal samples. The lack of high-depth whole genome sequencing (WGS) data means that the studies rely on imputation for SNPs and could be missing potential associations from insertions/deletions (indels), copy number variations (CNVs), especially for rare variants. In addition, previous GWAS studies for the gut microbiome investigated populations of European ancestry, how host genetics shape the gut microbiome in an Asian population remains unknown.

In this study, we identified genetic-microbial associations using for the first time highdepth sequencing data for both whole-genome and metagenome, in a high-depth discovery cohort of 632 healthy Chinese individuals and a low-depth validation cohort of 663 individuals. Prevotella and Bifidobacterium are the most heritable taxa after the cellulosedegrading phylum Fibrobacteres in this Chinese cohort, in agreement with twins results from European (Xie et al., 2016) as well as Asian (Lim et al., 2017) cohorts. With WGS data, we are uniquely positioned to comprehensively investigate common variants, rare variants, CNVs and the HLA (human leukocyte antigen) locus associated with the gut microbiome. Considering the reported gender differences in the gut microbiome (Xiao et al., 2016; Xiao et al., 2015) and increasing interest in incorporating the gender perspective into metagenomic and genomic studies (Khramtsova et al., 2018), we carry out the first gender-specific MGWAS to understand the difference of gut microbiome-genome association between genders. Together, our results reveal host genetic influences on the composition and functional potential of the gut microbiome to an unprecedented extent and generate a number of testable hypothesis for diseases such as colorectal cancer and cardiometabolic diseases.

\section{Results}

\section{Characteristics not reported in European cohorts}

To investigate genetic influences on the gut microbiome, we performed whole-genome 
sequencing on 632 blood samples to a mean depth of $44 \times$ (range from $32 \times$ to $52 \times$, Fig. S1A, Table S1A) per individual, and metagenomic sequencing on 632 stool samples to an average of $8.57 \pm 2.21 \mathrm{~GB}$ (Fig. S1B). This discovery cohort had a mean age of $30.7 \pm 5.5$ years (mean \pm s.d.; range of 6-35 years), a mean body mass index (BMI) of (21.8 \pm 6.3 ) and 53.5\% were female (Table S1B). We observed in this Chinese cohort that each genome differs between one another by 3.9 to 4.9 million sites (Table S1C). Variants were directly determined from the high-depth human genomes, including 38 million SNPs, 5 million indels, and 40 thousand CNVs. 6.5 million of these were common variants (minor allele frequency $(\mathrm{MAF})>0.05), 36.5$ million of these were rare and low-frequency variants $(\mathrm{MAF}) \leq 0.05$ ). Taxonomic profiling of the fecal metagenomes resulted in 19 phyla, 21 classes, 40 order, 77 families, 307 genera and 519 species. The top five abundant phyla in this cohort were Bacteroidetes (relative abundance of $51.0 \pm 13.5 \%$ ), Firmicutes $(11.2 \pm 5.6 \%)$, Proteobacteria $(2.8 \pm 3.7 \%)$, Fusobacteria $(0.3 \pm 1.1 \%)$ and Actinobacteria $(0.13 \pm 0.27 \%)$ (Fig. S2). Based on existing knowledge, we performed all M-GWAS by including covariates for gender, age, BMI, diet and lifestyle factors, stool form, defecation frequency, as well as the top four principal components to account for population structure (Table S1B; methods).

Unlike M-GWAS using chip data from European cohorts, we identified suggestive host genetic associations for the controversial concept of enterotypes (Fig. 1, Table S1D), possibly due to higher prevalence of Prevotella in developing countries (Dhakan et al., 2019; Vangay et al., 2018). Principle coordinate analysis (PCoA) as well as Dirichlet multinominal mixture model using Bray-Curtis dissimilarity showed that this Chinese cohort could be formed into two clusters dominated by Bacteroides and Prevotella (Zou et al., 2019), containing 440 and 178 individuals, respectively (Fig. 1A). The top two loci associated with the Bacteroides-Prevotella dichotomy $\left(P_{P-B}=2.08 \times 10^{-6}\right.$ and $P_{P-B}=2.6 \times 10^{-6}$, respectively, using Prevotella as cases and Bacteroides as controls in a logistic regression model) together explained $11 \%$ of the variance of the Bacteroides versus Prevotella 'enterotype'. Despite a report challenging the negative association between Bacteroides and Prevotella (Vandeputte et al., 2017), due to the statistically well-known loss of one degree of freedom in compositional data (Cao et al., 2019), genetic associations for these genera also showed the opposite trend. The minor allele of the top SNP, rs13045408 at BTBD3-LINC01722, positively correlated with Bacteroides abundance $\left(\beta=0.043 ; \mathrm{P}=5.3 \times 10^{-3}\right)$ and negatively correlated with Prevotella abundance $\left(\beta=-1.76 ; \mathrm{P}=1.6 \times 10^{-4}\right)\left(P_{P-B}=2.1 \times 10^{-6} ;\right.$ Fig. 1C); on the other hand, the minor allele of the other SNP rs1453213 at OXRI positively correlated with Prevotella $\left(\beta=2.23 ; \mathrm{P}=1.3 \times 10^{-7}\right)$ and negatively correlated with Bacteroides $(\beta=-$ $\left.0.049 ; \mathrm{P}=3.4 \times 10^{-4}\right)\left(P_{P-B}=2.6 \times 10^{-6}\right)$.

In order to validate these suggestive associations, we sequenced a validation cohort of 663 individuals (metagenomic shotgun sequencing for stool samples to an average of $8.59 \pm 2.14$ GB (Fig. S1D), but $7 \times$ whole-genome sequencing for human genome (range from $5 \times$ to $12 \times$, Table S1A; Fig. S1C)). Summary statistics of the covariates was largely similar (Table S1B). This replicate cohort comprised of 473 Bacteroides-dominant and 190 Prevotella-dominant individuals (Fig. 1B). The top 2 associations for the Bacteroides-Prevotella dichotomy remained (Fig. 1D; Table S1D; $P_{P-B}=0.024$ for rs1453213 in $O X R 1$ and $P_{P-B}=0.061$ for rs13045408 at BTBD3-LINC01722). 
We next investigated associations between genetic variation and microbiome $\beta$-diversity. This analysis found five loci with marginal genome-wide significance $\left(P<5 \times 10^{-8}\right.$; Fig. 1E; Table S1F). Three SNPs, rs60689247 in MAGI2, rs7716962 in XRCC4 and rs61823500 in Clorf21, were located in the intronic region of genes. MAGI2 related to multiple phenotypes or diseases in the GWAS catalog (MacArthur et al., 2017), including body mass index (BMI), schizophrenia, coronary artery calcification and type 2 diabetes, etc. The protein encoded by $X R C C 4$ functions together with DNA ligase IV and the DNA-dependent protein kinase in the repair of DNA double-strand breaks. The other two SNPs, rs11732767 and rs1967284 are located in the intergenic regions of $C D K N 2 A I P$ and $T B C 1 D 1$, respectively. CDKN2AIP is critical for the DNA damage response and TBC1D1 is link to Crohn's disease, lymphocyte count, etc. These are interesting associations, given the increasing incidences of Crohn's disease and cancer. The association between rs61823500 at Clorf21 and $\beta$-diversity could be replicated both in our replication cohort and German cohort $(P<0.05)$ (Wang et al., 2016). However, the three previous studies (Goodrich et al., 2016; Rothschild et al., 2018; Wang et al., 2016) identified a total of 64 SNPs associated with beta-diversity of gut microbiota. Of these, only one SNP was replicated here with nominal significance $(P=0.013$, Table S1G), and none was significant after multiple-test correction. 8 of the 64 SNPs were not found or rare in the Chinese population (MAF < 0.01). The allele frequencies of these 64 SNPs were significantly different between Chinese and European populations (T-test $P_{\text {difference }}=1.55 \times 10^{-}$

${ }^{5}$, Fig. S3). Besides, 380 SNPs have been previously reported to associate with specific taxa and we were able to replicate 11 of these $\left(P<0.05 / 380=1.3 \times 10^{-4}\right.$; Fig. 1F; Table S1H), especially in association with Bacteroides stercoris (Rothschild et al., 2018). 92 of the 380 loci were not found or rare in Chinese population. In summary, huge population heterogeneity exists, as is known from GWAS studies(Wojcik et al., 2019), and it is necessary to identify Asian-specific host genome-microbiome associations for better understanding genome-microbiome interactions among different ethnicities.

\section{Heritability estimates underscore genetic contributions to gut microbiome}

To assess the influence of host genetics on the gut microbiota, we calculated heritability estimates $\left(\mathrm{h}_{W G S}^{2}\right)$ for each specific taxon using whole-genome genetic variants. Among the 510 common taxa with at least $50 \%$ occurrence rate of all samples, 39 taxa $(7.6 \%)$ had a moderate or higher heritability greater than $0.6 ; 26$ taxa are significantly heritable with $\mathrm{h}_{W G S}^{2}>0.8$ and likelihood-ratio test (LRT) $P<0.05$ while evaluating with GREML-LDMS (Yang et al., 2015) (Fig. 2A and Table S2A). These heritable taxa mainly belong to the Bacteroidetes, Firmicutes and Actinobacteria phyla. Prevotella and Bifidobacterium, displayed the highest heritability at genus level with $\mathrm{h}_{W G S}^{2}=0.90$ and 0.88 , respectively (Fig. 2A). Similarly, their members, species Prevotella melaninogenica, Prevotella veroralis and Prevotella bryantii belonging to Prevotella, species Bifidobacterium longum and Bifidobacterium catenulatum-Bifidobacterium pseudocatenulatum complex belonging to Bifidobacterium, all showed high heritability $\left(\mathrm{h}_{W G S}^{2}>0.85\right.$, LRT $\left.P<0.05\right)$. Our results are consistent with the UK twins' (Xie et al., 2016) and Korean twins' studies (Lim et al., 2017) which reported that genus Prevotella $(\mathrm{h}=0.57)$ and Bifidobacterium $(\mathrm{h}=0.457)$ had high heritability, respectively. These were also heritable in the low-depth validation cohort 
$\left(\right.$ Prevotella $\mathrm{h}_{W G S}^{2}=0.87 ;$ LRT $\mathrm{P}=0.037$, and Bifidobacterium $\mathrm{h}_{W G S}^{2}=0.92 ;$ LRT $\mathrm{P}=0.030$, Table S2A).

Taking advantage of the metagenomic shotgun data, we further evaluated heritability of the functional capacity of the gut microbiome, according to the Kyoto Encyclopedia of Genes and Genomes (KEGG) orthologues (KOs) and gut microbial modules (GMMs) profiles. We identified 4919 common KOs and 98 common GMMs present in 50\% or more of the samples. Of which, 152 KOs and 12 GMMs showed high heritability $\left(\mathrm{h}_{W G S}^{2}=0.654 \sim 0.906\right.$, LRT $P<$ 0.05 , Fig. 2B, Table S2B,C). These heritable functional modules included carbohydrate metabolism (mannose degradation, fructan degradation, galactose degradation, rhamnose degradation and maltose degradation), organic acid metabolism (butyrate production, lactaldehyde degradation and formate conversion) and lipid metabolism (glycerol degradation). Among the 152 heritable KOs, $41 \mathrm{KOs}$ were enriched in gut microbiome data of type 2 diabetes (T2D) patients compared to non-diabetic controls (Qin et al., 2012). Of which, aldehyde reductase (K00011) and PTS system, galactitol-specific IIB component (K02774) belonged to the galactose metabolism pathway (ko00052); pyruvate, orthophosphate dikinase (K01006) belonged to the pyruvate metabolism pathway (ko00620). These two KEGG pathways were previously reported to be associated with T2D risk by genome-wide association study (Perry et al., 2009). Furthermore, three KOs, including two T2D-associated KOs K0008 and K01138, and K04075 were confirmed as heritable in the replication cohort $\left(\mathrm{h}_{W G S}^{2}=0.798 \sim 0.863\right.$, LRT $P<0.05$; Table S2B). We also confirmed one heritable GMM, i.e. butyrate production I $\left(\mathrm{h}_{W G S}^{2}=0.872\right.$, LRT $P=0.049$; Table S2C), consistent with twins' data from UK (Xie et al., 2016). These evidences suggest that some of the heritable KOs in the gut microbiome were determined by host genetic variants and may increase the risk of metabolic diseases such as T2D.

Next, we investigated the genetic variants correlated with functional capacity of the gut microbiome according to GMMs. We found eight loci significantly associated with seven GMMs $\left(P<5 \times 10^{-8}\right.$; Table S2D). The strongest association was identified for maltose degradation $\left(\mathrm{h}_{W G S}^{2}=0.65\right)$ and nine SNPs $\left(P=4.5 \times 10^{-9}\right)$ at SLC41A2 gene which involved in transport of glucose and other sugars, bile salts and organic acids, metal ions and amine compounds. We also found genetic signals for butyrate production $\left(\mathrm{h}_{W G S}^{2}=0.89\right)$ and mucin degradation $\left(\mathrm{h}_{W G S}^{2}=0.63\right)$. Mucin degradation has been implicated with metabolic regulation, obesity and type 2 diabetes. Three SNPs near CCR3 gene associated with mucin degradation and $C C R 3$ gene was reported as enhancer of BMI-related phenotypes and coronary artery disease $\left(P<1.0 \times 10^{-8}\right)$ in transcriptome-wide association study (Gusev et al., 2016), and our results suggest that mechanistic investigations of these SNPs should take the gut microbiome into consideration.

\section{Common variants M-GWAS identifying abundant genetic signals for gut microbiome}

To detect associations between gut microbial taxa and specific genetic variants, we at first performed common variants M-GWAS analysis using a linear model for taxa present in over 95\% of individuals and a logistic model for zero-inflated taxa (Table S3A). We identified 
169347 significant associations involving 37 loci and 51 bacterial taxa $\left(P<5 \times 10^{-8}\right.$; Fig. 3A,

170 Table S3B $)$. The strongest signal $\left(P=1.68 \times 10^{-9} ;\right.$ Fig. S4A,B $)$ was observed for the phylum 171 Actinobacteria and its members, which is consistent with their high heritability (Fig. 2A). 172 Actinobacteria associated with SNP rs62183161 at LOC150935 gene, which had been 173 reported linked to body composition measurement and energy intake (Comuzzie et al., 2012). 174 Similarly, the other highly heritable taxa, family Prevotellaceae was associated with SNP 175 rs1453123 in OXRI (oxidation resistance 1; $P=1.58 \times 10^{-8}$; Fig. S4C,D) gene, the protein 176 encoded by which controls the sensitivity of neuronal cells to oxidative stress and lacking Oxr1 caused cerebellar neurodegeneration in mice (Oliver et al., 2011). Eggerthella abundance was associated with a missense variant rs3749147 in the GPN1 gene $(P=3.2 \times$ $\left.10^{-8}\right)$. Rs 3749147 had been reported to associate with waist circumference - triglycerides in GWAS catalog and GPN1 gene is linked to oral cavity cancer, palmitoleic acid levels and periodontitis. Seven of the 37 genome-wide significant loci have been reported to associate with traits or diseases in the GWAS catalog (Table S3C). We were able to replicate 13 of the 37 associations in the low-depth validation cohort; rs13420238 at LOC150935 had a $P=$ 0.007 with Bifidobacterium longum, rs1453123 at $O X R 1$ had a $P=0.006$ with Prevotellaceae and rs760646544 near PLXDC2 have a $P=0.043$ with Roseburia intestinalis (Fig. 3B,C,D; Table S3B).

Furthermore, functional annotations using FUMA (Watanabe et al., 2017) tool showed the 37 loci were mapped to 109 genes which were involved in three main traits and diseases in GWAS catalog (MacArthur et al., 2017) (false discovery rate (FDR) adjusted $P<0.05$, Fig. 3E) : (1) metabolism related traits: waist circumference - triglycerides, metabolite levels (homovanillic acid), serum uric acid levels in response to allopurinol in gout, acute insulin response, fasting blood glucose, total cholesterol levels and HDL cholesterol levels; (2) immune-related diseases: Inflammatory skin disease, systemic lupus erythematosus and type 2 diabetes. (3) nervous system related disease: Alzheimer's disease (cognitive decline) and loneliness. Meanwhile, we perform gene set enrichment analyses and identified 16 significantly enriched KEGG or GO terms after FDR correction $(P<0.05$, Fig. 3F), including multiple metabolic process such as propanoate, fatty acid, diacylglycerol and alditol metabolism, as well as neutral lipid biosynthetic process.

\section{Rare variants- and CNVs-based M-GWAS further revealed genetic contributions to gut microbiome}

Given the advantages of high-depth WGS and that $85 \%(36.5 \mathrm{M} / 43 \mathrm{M})$ of the variants were rare (MAF <0.05), we globally considered whether rare variants in any gene and copy number variants contributed to the gut microbiome composition. We tentatively identified 60 associations involving 47 genes and 54 bacterial taxa $\left(\mathrm{P}<2.14 \times 10^{-6}=0.05 / 27874\right.$ for Bonferroni correction of 27,874 individual genes; Table S3D), e.g. PCSK9 gene, a popular target for lowering LDL cholesterol. We evaluated the interaction between proteins encoded by 47 genes by constructing Protein-protein interaction (PPI) networks. We found there were 34 proteins participating in the connected network among the 47 genes (Fig. S5). The 34 proteins have more interactions than expected for a random set of proteins of similar size 
211 (enrichment $P=0.037$ ), indicating functional intersection of the 34 microbiome-associated 212 proteins. KEGG pathway analysis of the 34 genes showed enrichment in 2 main pathways 213 (Table S3E), including hsa03030:DNA replication (FDR=0.002) and hsa01100:Metabolic 214 pathways $(\mathrm{FDR}=0.044)$.

215 CNVs-based M-GWAS identified 18 significant CNVs associated with 20 bacterial taxa (P $<6.25 \times 10^{-6}=0.05 / 8000$ for Bonferroni correction of $8 \mathrm{~K}$ common CNVs with MAF $>0.01$, Table S3F). Of which, 13 CNVs overlaps with reported CNVs recorded in the Database of 218 Genomic Variants (DGV) (MacDonald et al., 2014). Eight CNVs resides within the genes 219 mainly in the intronic region. We identified the species butyrate-producing bacterium SS3/4 220 associated with a $28.7 \mathrm{~kb}$ CNV region (chr4:69384168-69412841; $P=3.1 \times 10^{-6}$, frequency $=$ 0.03 ) which located $67 \mathrm{~kb}$ downstream of the UGT2B4 gene. The CNV includes many variants involved in expression quantitative trait loci (eQTL) and regulate the expression of $U G T 2 B 4$ in heart tissue and UGT2B28 in the Esophagus Mucosa and liver (Table S3G), consistent with functions of butyrate or other bacterial metabolites in these tissues. Moreover, one SNP rs12505338 and the other three SNPs in the CNV region were associated with serum concentration of stearate $(18: 0)\left(P=9.3 \times 10^{-5}\right.$, Table S3F $)$ and glutamate $(P=7.3 \times$ $10^{-6}$ ), respectively, by looking for the NHLBI GRASP catalog (Leslie et al., 2014). Thus, associations with the gut microbiome due to rare variants and CNVs also have important functional implications for host physiology.

Common variants-, rare variants- and CNVs- based associations separately explained $8.3 \%$, $11.4 \%$ and $4.9 \%$ of the microbiome composition, respectively. In addition, the average occurrence frequency of gut microbiome taxa associated with common variants or rare variants were 0.768 and 0.596, respectively (Tables S3B and S3D; Wilcoxon test, $\mathrm{P}=0.008$ ). These results indicate that host rare variants also shaped the gut microbiome, especially for less common members of the community.

\section{A gene-bacteria axis in gender-differential metabolic and neuronal functions}

$53.5 \%$ of this cohort were female, permitting a comparison between genders. Females showed higher alpha diversity than men (Wilcoxon test, $\mathrm{P}<0.05$; Fig. S6), and we identified 32 significantly different taxa between genders in discovery cohort and 27 of which were consistent in replication cohort (FDR q <0.1 using MaAslin; Table S4A). Phylum Actinobacteria and its members, including class Actinobacteria, order Bifidobacteriales, family Bifidobacteriaceae, genus Bifidobacterium were all significantly enriched in females. However, Fusobacterium was significantly enriched in male.

Since the gut microbiome exhibited striking difference between males and females, we performed a gender stratified association analysis between host genetic variants and gut bacteria, the 37 associations were overlapped between genders (Table $\mathbf{S 4 B}, \mathrm{P}<0.05$ both in males and in females, and $\mathrm{P}<5 \times 10^{-8}$ in combined results), identical to the combined analysis (Table S3B.). Especially, we identified 33 male-specific $\left(\mathrm{P}<5 \times 10^{-8}\right.$ in males but $\mathrm{P}>0.05$ in females) and 37 female-specific associations ( $\mathrm{P}<5 \times 10^{-8}$ in females but $\mathrm{P}>0.05$ in males) linked to gut bacteria (Fig. 4A and Table S4C). We compared the effect sizes of identified 
252

253

254

255

256

257

258

259

260

261

262

263

264

265

266

267

268

269

270

271

272

273

274

275

276

277

278

279

280

281

282

283

284

285

286

287

288

289

290

291

292

\section{3}

variants between genders, and confirmed that all the variants showed a significant difference ( $P_{\text {difference }}<0.01$ ). Five loci of the 70 associations linked to traits or diseases in GWAS catalog (Table S4D). Rs4650205 at NEGRI-LINC01360 gene significantly associated with the abundance of genus Acidaminococcus in males $\left(P=4.87 \times 10^{-8}\right)$ but not in females $(P=$ 0.48 ), and its proxy SNPs (linkage disequilibrium $r^{2}>0.6$ ) were reported linked to multiple nervous-system disorders such as autism spectrum disorder, schizophrenia, depression and migraine by substantial GWAS studies. Female-specific SNP rs61781314 at LEPR gene was associated with both genus Eggerthella and species Eggerthella lenta, and its proxy SNP rs17415296 linked to blood protein levels $\left(P=4 \times 10^{-229}\right)$. Eggerthella lenta as an opportunistic pathogen have been reported to underlie human infections and enriched in T2D (Qin et al., 2012), rheumatoid arthritis (RA) (Zhang et al., 2015) and atherosclerotic cardiovascular disease (ACVD) patients (Jie et al., 2017). The protein encoded by LEPR is a receptor for leptin (an adipocyte-specific hormone that regulates body weight), and is also involved in the regulation of fat metabolism and pituitary dysfunction. In the low-depth validation cohort (327 males and 336 females), associations including the male-specific association of rs6871146 with Lactococcus $\left(\beta_{\text {male }}=0.42\right.$ and $P_{\text {male }}=0.027 ; \beta_{\text {male }}=-0.47$ and $\left.P_{\text {female }}=0.062\right)$ and the female-specific association of rs7165633 and Mobiluncus mulieris $\left(\beta_{\text {male }}=0.009\right.$ and $P_{\text {male }}=0.96 ; \beta_{\text {female }}=-0.48$ and $\left.P_{\text {female }}=0.008\right)$ were replicated. The femalespecific association with Mobiluncus suggests an intestinal reservoir for the bacterium which is involved in vaginal infections (Onderdonk et al., 2016).

We investigated the overlapped genes between gender-specific genes and traits or diseasesassociated genes in GWAS catalog (Fig. 4B-E), then found that genes located in femalespecific loci enriched in four phenotypes, including two metabolic traits, i.e. metabolite levels (HVA) and C-reactive protein levels or triglyceride levels (pleiotropy). Genes located in male-specific loci enriched in mainly the systemic lupus erythematosus related traits. Interestingly, one locus chr19:53772987- 53796549 was both male and female-specific locus although associated with different taxa in different genders, and this locus located nearby the gene family MIR371A-MIR372-MIR373. WikiPathway analysis showed this gene family related to "miRNAs involved in DNA damage response", suggesting that gut bacteria may participated in DNA damage response in both genders. Additionally, gender-specific loci were also enriched in the pathway "leptin insulin overlap", consistent with the association between LEPR gene and Eggerthella lenta as described above. Moreover, gene set enrichment analysis identified 37 female-specific loci involved in pathways "olfactory signaling" and "signaling by G-protein-coupled receptor (GPCR)", females had been reported superior to males in olfactory abilities (Brand and Millot, 2001) and had higher levels of Gprotein-coupled kinases [GPCR kinase (GRK)] 3 and 5 than male(Bychkov et al., 2011). Male-specific loci related to pathways "systemic lupus erythematosus" and "leishmania Infection". Taken together, the gut microbiome exhibited differential associations with the human genome in males and females, and might contribute to different metabolic and neuronal functions as well as disease susceptibility.

\section{M-GWAS helps understand biomarkers from MWAS}


We note that our M-GWAS discovered signals for some of the bacteria often reported from metagenome-wide association studies (MWAS) (Wang and Jia, 2016), e.g. three butyrateproducing species, Roseburia intestinalis, Eubacterium rectale and Faecalibacterium prausnitzii, have been associated with healthy controls in MWAS for T2D, ACVD and obesity as well as Alistipes shahii associated with lower BMI (Jie et al., 2017; Liu et al., 2017; Qin et al., 2012)(Fig. 5A). We observed the species Roseburia intestinalis associated with rs760646544 (a insertion of CTGTT) near PLXDC2 $\left(P=1.75 \times 10^{-8}\right.$, related to nidogen-1 measurement and diabetic retinopathy in GWAS catalog). Species Eubacterium rectale negatively associated with rs1555188 near PHF21B in females $\left(P=4.52 \times 10^{-9}\right)$ but not in males $(P=0.55)$. Genus Faecalibacterium and species $F$. prausnitzii were identified linked to DYNLL1 gene $\left(P=8.83 \times 10^{-8}\right)$ which included 94 rare variants in gene-based association analysis. Alistipes shahii associated with rs72627489 near SOWAHC in gender-combined analysis $\left(P=8.58 \times 10^{-9}\right)$ and rs914338 near $U N C 93 A$ in male-specific analysis $(P=2.40$ $\left.\times 10^{-8}\right)$. We confirmed the association between Roseburia intestinalis and rs760646544 (a insertion of CTGTT) near PLXDC2 in replicate cohort $(P=0.043$; Fig. 3D; Table S3B). Consistently, the abundance of $R$. intestinalis showed higher correlation in monozygotic compared to dizygotic twins from the United Kingdom(Xie et al., 2016).

Bifidobacterium dentium, enriched in RA (Zhang et al., 2015), ACVD as well as schizophrenia patients. CNVs-based M-GWAS analysis identify the association between Bifidobacterium dentium and nucleoredoxin $(N X N)$ with copy loss of 642 bp (chr17:898377899018; $P=4.11 \times 10^{-6}$; Fig. 5B), and nucleoredoxin 1 as the oxidoreductase protects antioxidant enzymes such as catalase from ROS-induced oxidation in plant cells (Kneeshaw et al., 2017). In addition, $N X N$ was significantly high expressed in normal tissue samples compared with colon adenocarcinoma (COAD) and rectum adenocarcinoma (READ) cases (Fig. 5C). Similarly, Parvimonas micra is enriched in CRC (Wang and Jia, 2016), its associated host gene PARVB (Parvin Beta; rs35928604; $P=1.55 \times 10^{-8}$; Fig. 5D) was overexpressed in CRC including COAD and READ (Fig. 5E), which supported the previous study that reported overexpression of $P A R V B$ correlated significantly with lymph node metastasis and tumor invasion (Bravou et al., 2015). Bifidobacterium dentium, Parvimonas micra and Porphyromonas asaccharolytica are all bacteria found in the human oral cavity that are normally in low abundance in the colon. These findings are consistent with the notion that immune defense are important drivers of host-microbiome co-evolution in addition to metabolism.

\section{Discussion}

The present study is the first complete and comprehensive genome-wide M-GWAS analysis integrating a total of 1295 host whole genome and fecal whole metagenome sequencing to investigate the associations between genetic variants and gut microbiome in Chinese adults. Using common variants-, rare variants-, and CNVs-based association analysis without loosening the p-value cutoff, we identified 37 loci, 47 genes and $20 \mathrm{CNVs}$ 
335

significantly associated with gut bacterial taxa, and they additively explained no less than $20 \%$ of the microbiome composition. Although study-wise significant associations would be more desirable ( $\mathrm{P}<1 \times 10^{-10}$ for over 500 taxa), consistent with previous M-GWAS from Germany, the Netherlands and Israel(Bonder et al., 2016; Rothschild et al., 2018; Wang et al., 2016), abundant signals were only detected with genome-wide significance. Notably, although insufficient power to detect variants (rare variants and CNVs etc.) in low-depth sequencing data, we still replicated our key findings for 'enterotypes', T2D-KOs, common variants' associations, gender-differential associations, and MWAS markers (Figs. 1, 3, 6, Table S3B, S4C). The majority of associations lie in metabolic, neurological and immunological functions, which is particular interesting considering the rapid changes in lifestyle and environmental factors in China and the rising disease incidences. For example, a good portion of our Chinese cohort still harbor Prevotella instead of Bacteroides, compared with western country (Vangay et al., 2018). To investigate the effect of host genome on enterotype, we identified two suggestive loci explaining $11 \%$ of the Prevotella-Bacteroides variances. These two tentative associations are not yet genome-wide significant $\left(P_{P-B}=2.08 \times 10^{-6}\right.$ and $P_{P-B}=$ $2.6 \times 10^{-6}$, respectively, using Prevotella as cases and Bacteroides as controls in logistic regression model), but we feel obliged to report them after validation, given the long-lasting arguments in multiple studies(Costea et al., 2018; Jeffery et al., 2012; Knights et al., 2014) over the concept of 'enterotypes'. It is intriguing that heterozygous individuals show two clusters of either high or low Prevotella (Fig. 1). In addition, we identified heritability and specific loci for Prevotella species; the minor allele T of rs 1453213 at OXR1 was consistently correlated with higher abundance of family Prevotellaceae and Prevotella species, and higher frequency of allele $\mathrm{T}$ in Asian population ( $\mathrm{f}=0.39$ ) than European population $(\mathrm{f}=0.28)$ may also explain the enrichment of Prevotella in Asian in addition to the diet. More cohorts from developing countries in the future with a higher fraction of Prevotella-dominated individuals would help further confirm these results.

Due to the emphasis on diet in early studies and recently on medication, metagenome-wide association studies (MWAS) (Qin et al., 2012; Wang and Jia, 2016) have received even more controversy than GWAS. Besides diet and medication, we also took into account physical activity in this 4D-SZ cohort (Jie et al., 2019a; Jie et al., 2019b). Here we find that fecal biomarkers previously reported by MWAS studies on colorectal cancer and metabolic diseases have some associations with host genetics, while some taxa especially some sporeforming bacteria lacked host genetic associations. With 1 liter of saliva swallowed every day, genetically encoded responses to ectopic presence of oral bacteria in the gut may be a common theme in a number of diseases investigated by MWAS, as has been shown for inflammatory bowel disease (Atarashi et al., 2017).

Gender stratification GWAS could be used to identify novel loci that may have been previously undetected in gender-combined GWAS and had been performed in human complex traits(Khramtsova et al., 2018; Zeng et al., 2018), while none had done it for gut microbiome. Here, we performed the first gender-specific M-GWAS and identified 33 malespecific (involving in inflammation, such as SLE and leishmania infection) and 37 femalespecific associations (involving in olfactory signaling and GPCR signaling) linked to gut bacteria by gender-specific analysis, suggesting the importance of discriminating gender in M-GWAS studies and it will help better understand the underlying molecular mechanisms 
379

386 between genders. In summary, our results unveil host genetic influence on the gut microbiome to an unprecedented extent and first reveal the influence of host genome on gender-differential gut bacteria.

\section{STAR Methods}

\section{KEY RESOURCES TABLE}

\begin{tabular}{lll}
\hline Reagent or Resource & Source & Identifier \\
\hline Blood samples & This paper & N/A \\
Fecal samples & This paper & N/A \\
\hline
\end{tabular}

Deposited Data

Raw sequencing data This paper

CNGB database accession CNP0000289

Software and Algorithms

Whole genome sequencing data

\begin{tabular}{|c|c|c|}
\hline GATK & McKenna et al., 2010 & https://software.broadinstitute.org/gatk/ \\
\hline SpeedSeq & Chiang et al., 2015 & https://github.com/hall-lab/speedseq \\
\hline PLINK & Purcell et al., 2007 & http://zzz.bwh.harvard.edu/plink/plink2.shtml \\
\hline GCTA & Yang et al., 2015 & http://cnsgenomics.com/software/gcta/\#Overview \\
\hline FUMA & Watanabe et al., 2017 & http://fuma.ctglab.nl/ \\
\hline GEPIA & Tang et al., 2017 & http://gepia.cancer-pku.cn/ \\
\hline \multicolumn{3}{|c|}{ Metagenome sequencing data } \\
\hline IGC & Li et al., 2014 & http://meta.genomics.cn/meta/home \\
\hline GMM & Vieira-Silva et al. 2016 & https://github.com/raeslab/GMMs \\
\hline microbiomeGWAS & Xing Hua, 2015 & https://github.com/lsncibb/microbiomeGWAS \\
\hline $\mathrm{R}$ & $\begin{array}{l}\text { R Project for Statistical } \\
\text { Computing }\end{array}$ & http://www.R-project.org/ \\
\hline
\end{tabular}

LEAD CONTACT FOR REAGENT AND RESOURCE SHARING

Further information and requests for resources and reagents should be directed to and will be 
393

394

395

396

397

398

399

400

401

402

403

404

405

406

407

408

409

410

411

412

413

414

415

416

417

418

419

420

421

422

423

424

425

426

427

428

429

430

431

432

433

434

435

436

437

\section{EXPERIMENTAL MODELS AND SUBJECT DETAILS}

\section{Cohort descriptions}

632 individuals were enlisted in the discovery cohort and 663 individuals were enlisted in the replication cohort, as part of the larger effort of 4D-SZ study(Jie et al., 2019a; Jie et al., 2019b). Questionnaires were collected through a cell phone application. After excluding individuals that were pregnant, taking antibiotics within one month or suffering from diseases, 620 individuals in the discovery cohort and 663 individuals in the replicate cohort were remained. All participants provided blood samples during physical examination. The MGIEasy stool collection kit containing a room temperature stabilizing reagent that preserves metagenomic samples (Han et al., 2018), were also given to the volunteers, who handed in fecal samples on the same morning or the day after. All samples were retrieved from the boxes in front of restrooms and then stored at $-80^{\circ} \mathrm{C}$ before DNA extraction. For blood sample, buffy coat was isolated and DNA was extracted using HiPure Blood DNA Mini Kit (Magen, Cat. no. D3111) according to the manufacturer's protocol. Feces were collected by MGIEasy and stool DNA was extracted in accordance with the MetaHIT protocol(Qin et al., 2012) as described previously. The DNA concentrations from blood and stool samples were estimated by Qubit (Invitrogen). $200 \mathrm{ng}$ of input DNA from blood and stool samples were used for library formation and then processed for single-end 100bp sequencing on BGISEQ500 platform(Fang et al., 2018).

The study was approved by the Institutional Review Boards (IRB) at BGI-Shenzhen, and all participants provided written informed consent at enrolment.

\section{METHOD DETAILS}

\section{High-depth WGS alignment and SNP/indel calling in discovery cohort}

Whole-genome reads were aligned to latest reference human genome GRCh38/hg38 with BWA(Li and Durbin, 2009) (version 0.7.15) with default parameters. The reads consisting of base quality $<5$ or containing adaptor sequencing were filtered out. The alignments were indexed in the BAM format using Samtools (Li et al., 2009a) (version 0.1.18) and PCR duplicates were marked for downstream filtering using Picardtools (version 1.62). The Genome Analysis Toolkit's (GATK(McKenna et al., 2010), version 3.8) BaseRecalibrator created recalibration tables to screen known SNPs and INDELs in the BAM files from dbSNP (version 150). GATKlite (v2.2.15) was used for subsequent base quality recalibration and removal of read pairs with improperly aligned segments as determined by Stampy. GATK's HaplotypeCaller were used for variant discovery. GVCFs containing SNVs and Indels from GATK HaplotypeCaller were combined (CombineGVCFs), genotyped (GenotypeGVCFs), variant score recalibrated (VariantRecalibrator) and filtered (ApplyRecalibration). During the GATK VariantRecalibrator process, we took our variants as inputs and used four standard SNP sets to train the model: (1) HapMap3.3 SNPs; (2) dbSNP build 150 SNPs; (3) 1000 Genomes Project SNPs from Omni 2.5 chip; and (4) 1000G phase1 high confidence SNPs. The sensitivity threshold of $99.9 \%$ to SNPs and $99 \%$ to indels were applied for variant selection after optimizing for Transition to Transversion (TiTv) ratios using the GATK ApplyRecalibration command. After applying the recalibration, there are 43,342,216 raw variants left, including 38 million SNPs, 5 million indels. 
We applied a conservative inclusion threshold for variants: (i) mean depth $>8 \times$; (ii) HardyWeinberg equilibrium (HWE) $P>10^{-4}$; and (iii) genotype calling rate $>98 \%$. We demanded samples to meet these criteria: (i) mean sequencing depth $>20 \times$; (ii) variant call rate $>98 \%$; (iii) no population stratification by performing principal components analysis (PCA) analysis implemented in PLINK(Purcell et al., 2007) (version 1.07) and (iv) excluding related individuals by calculating pairwise identity by descent (IBD, Pi-hat threshold 0.1875) in PLINK. Only 2 samples were removed in quality control filtering and 618 individuals entered into subsequent analysis.

\section{CNV calling}

448 The CNV call set were produced using the SpeedSeq(Chiang et al., 2015) pipeline, followed 449 by the svtools package (v0.2.0; https://github.com/hall-lab/svtools). Briefly, speedseq sv, 450 which comprises LUMPY for SV calling based on discordant pairs and split-reads; svtyper 451 for SV genotyping; and cnvnator for read-depth based CNV detection; was run on each 452 sample individually. The individual-level calls were sorted and merged using svtools lmerge, 453 and then each sample was re-genotyped and copy number annotated at all variant positions 454 using svtools genotype and copynumber, and pasted into a single cohort-level VCF. For filtering, inversion calls and adjacencies (i.e. BNDs) were excluded. The CNV was defined as known in the Database of Genomic Variants (DGV) (MacDonald et al., 2014) (http://projects.tcag.ca/variation) if it had $70 \%$ region overlapped with one CNV in DGV.

\section{Low-depth WGS alignment and SNP/indel calling in replicate cohort}

460 We used BWA to align the whole genome reads to GRCh38/hg38 and used GATK to 461 perform variants calling by applying the same pipelines for high-depth WGS data. After 462 finishing the GenotypeGVCFs process, we got 29906793 raw variants. A more stringent process in the GATK VariantRecalibrator stage compared with high-depth WGS was then used, as are recommended for low-coverage whole-genome data, to filter the uncertain genotype calls and keep only high-quality variants. Specifically, we excluded SNPs with low mapping quality $(\mathrm{Q}<20)$ and SNPs with low depth $(\mathrm{DP}<3)$. Further, we kept variants with less than 30\% missing information, leaving 779521 highly reliable variants. All these highquality variants were then imputed using BEAGLE 6(Browning et al., 2018) with 618 highdepth WGS dataset as reference panel. We retained only variants with imputation info. > 0.7 and got 5318809 imputed variants. Finally, we further filtered this set to keep variants with variants for subsequent analysis.

To evaluate the data quality, we sequenced 27 samples with both high-depth and low-depth WGS data and then compared the 5318809 variants between them for each individual. The average genotype concordance was $98.66 \%$ (Table S1I).

\section{Metagenomic sequencing and profiling}

The high-quality metagenomic sequencing reads were aligned to hg38 using SOAP2(Li et al., 2009 b) (version 2.22 ; identity $\geq 0.9$ ) to remove human reads. The gene profiles were generated by aligning high-quality sequencing reads to the integrated gene catalog (IGC)(Li et al., 2014) by using SOAP2 (identity $\geq 0.95$ ) as previously described. The relative abundance profiles of phylum, order, family, class, genera, species and Kyoto Encyclopaedia 
of Genes and Genomes (KEGG)(Kanehisa et al., 2014) orthologous groups (KOs) were determined from the gene abundances. To eliminate the influence of sequencing amount in comparison analyses, we downsized the unique IGC mapped reads to 20 million for each sample. The relative abundance profiles of gene, phylum, order, family, class, genus, species and KOs were determined accordingly using the downsized mapped reads per sample.

GMMs (gut metabolic modules) reflect bacterial and archaeal metabolism specific to the human gut, with a focus on anaerobic fermentation processes(Vieira-Silva et al., 2016). The current set of 103 GMMs was built through an extensive review of the literature and metabolic databases, inclusive of MetaCyc(Caspi et al., 2014) and KEGG, followed by expert curation and delineation of modules and alternative pathways. And we identified 98 common GMMs present in $50 \%$ or more of the samples.

\section{Covariates used in this study}

As part of the 4D-SZ cohort, all participants in this study had records of multi-omics data, including anthropometric measurement, stool form, defecation frequency, diet, lifestyle, blood parameters, hormone, etc. (Jie et al., 2019b). We tested for associations between these environmental factors and microbiome $\beta$-diversity at the genus level. The effect size and significance of the mentioned variables were estimated using 'envfit' function in vegan ( $R$ 3.2.5, vegan package 2.4-4). Gender, BMI and defecation frequency were identified to be the strongest factors to explain gut microbiome composition. They accounted for $3.79 \%, 2.14 \%$ and $1.79 \%$ of the microbiome variance, respectively. In addition, given the effects of diet and lifestyles on specific taxa, we finally included age, gender, BMI, defecation frequency, stool form, 12 diet and lifestyle factors, as well as the top four principal components (PCs) as covariates for all subsequent M-GWAS analysis (Table S1B).

\section{Enterotype analysis}

The enterotypes analysis was performed using genus-level gene abundance data according to the Dirichlet multinomial mixtures (DMM) based clustering approach(Ding and Schloss, 2014; Holmes et al., 2012) and two enterotypes were identified among the 618 healthy Chinese individuals in discovery cohort, including Bacteroides (enterotype 1, $\mathrm{n}=440$ ) and Prevotella (enterotype 2, $\mathrm{n}=178$ ). Using the same method, this replicate cohort comprised of 473 Bacteroides-dominant and 190 Prevotella-dominant individuals. We used logistic model implemented in PLINK to run a GWAS for genetic variation and the enterotype phenotype (ie. Bacteroides and Prevotella; dichotomous trait). We estimated the proportion of enterotypes' variance explained by top two loci using the restricted maximum likelihood (REML) method implemented in GCTA.

\section{Association analysis for microbiome $\beta$-diversity}

The microbiome $\beta$-diversity (between-sample diversity) based on genus-level abundance data were generated using the 'vegdist' function (Bray-Curtis dissimilarities). Then, we performed principal coordinates analysis (PCoA) based on the calculated beta-diversity dissimilarities using the 'capscale' function in 'vegan'. The associations between genetic variants and microbiome $\beta$-diversity was performed using microbiomeGWAS(Xing Hua, 2015) tool. 
528

529

530

531

532

533

534

535

536

537

538

539

540

541

542

543

544

545

546

547

548

549

550

551

552

553

554

555

556

557

558

559

560

561

562

563

564

565

566

567

568

569

570

571

\section{WGS-based heritability for gut microbiome}

Heritability was estimated with the GREML-LDMS(Yang et al., 2015) method implemented in GCTA(Yang et al., 2011) software. The GREML-LDMS method is proposed to estimate heritability using whole genome sequence (WGS) data. The method is unbiased and corrects for the LD bias in the estimated SNP-based heritability. The analysis involves four steps: 1) calculating segment-based LD score; 2) stratifying SNPs based on the segment-based LD score (this is done in R); 3) computing genotypic relatedness matrix (GRM) using the stratified SNPs; 4) performing REML analysis using the multiple GRMs. Therefore, the heritability $\mathrm{h}_{g}^{2}$ is then the fraction of the variance accounted for by the genetics and can be formulated as: $h_{g}^{2}=\operatorname{var}(g) / \operatorname{var}(g)+\operatorname{var}(e)$, where $\operatorname{var}(g)$ and $\operatorname{var}(\mathrm{e})$ are the genetic and residual variance components estimated by the REML approach.

In the present study, we estimate heritability $\mathrm{h}_{W G S}^{2}$ for the relative abundances of each taxon, KO and GMM conditioned on age, gender, BMI, defecation frequency, stool form, self-reported diet, lifestyle factors and top four PCs. We then defined the taxa, KOs and GMMs with LRT $\mathrm{P}<0.05$ in GREML-LDMS as significantly heritable.

\section{Genome-wide Association analysis for gut bacteria}

We tested the associations between host genetics and gut bacteria using linear or logistic model based on the abundance of gut bacteria. The abundance of bacteria appeared in over $95 \%$ of individuals was transformed by the natural logarithm and the outlier individual who was located away from its mean by more than five standard deviations was removed, so the abundance of bacteria could be treated as quantitative trait. Otherwise, we dichotomized bacteria into presence/absence patterns to prevent zero inflation, then the abundance of bacteria could be treated as dichotomous trait. Next, for the common variants with MAF > $5 \%$, we performed a standard single variant (SNP/indel)-based GWAS analysis via PLINK using a linear model for quantitative trait or a logistic model for dichotomous trait, a threshold of $P<5 \times 10^{-8}$ was used for genome-wide significance. We used the same methods for CNVs-based association analysis and set a significance threshold at $P<6.25 \times 10^{-6}$ accounting for 8,006 common CNVs (MAF > 1\%). For rare variants-based association analysis, we applied the Sequence Kernel Association Test(Wu et al., 2011) (SKAT) to the rare variants $(\mathrm{MAF} \leq 5 \%)$ for each gene. Gene regions were annotated using the RefSeq(Pruitt et al., 2012) database with a total of 27,874 genes. We only included the genes which had five or more rare variants (as recommended by the SKAT authors) for testing; 22,015 genes satisfied this requirement. Associations were considered significant with $\mathrm{p}<$ $2.14 \times 10^{-6}$ (equal to $0.05 / 22,015$ ). When testing all the association analysis, we adjusted for gender, BMI, defecation frequency, stool form, self-reported diet, lifestyle factors and the first four PCs.

The additive effect of the significant loci from this analysis was then determined using redundancy analysis based on genus-level composition ('rda' in the 'vegan' package) and the 'ordiR2step' function in the 'vegan' package in R. To quantify the fraction of microbiome variance that could be inferred from gene-based analysis (actually rare variants), we first selected 200 top-ranking rare variants (not in linkage equilibrium) according to their association with taxa, then performed a greedy stepwise algorithm, in which at each iteration we added the most significant variant to the inferred variant sets added in previous iterations. 
572 Before adding each variant, we performed 1000 permutation tests and verified that its 573 contribution was greater than in at least $50 \%$ of these permutations. If not, we stopped the 574 algorithm. In each permutation we assigned the top 200 rare variants of each individual to a 575 random individual, and then reran the entire analysis. Finally, 37 loci from common variants576 based association analysis, 76 loci from rare variants-based association analysis and 22 loci 577 from CNVs-based association analysis were used to infer the variance, respectively, and then 578 in combination.

\section{Functional annotation of significant loci}

582

Genome-wide significant loci identified in M-GWAS analysis were mapped to genes using SNP2GENE in FUMA(Watanabe et al., 2017) (http://fuma.ctglab.nl/). We first converted the loci positions from hg38 to hg19, then used the positional mapping method and maps variants to genes based on physical distance within a $20 \mathrm{~kb}$ window. Mapped genes were further investigated using the GENE2FUNC procedure, which provides hypergeometric tests of enrichment of the list of mapped genes in 53 GTEx tissue-specific gene expression sets, 7,246 MSigDB gene sets, and 2,195 GWAS catalog(MacArthur et al., 2017) gene sets. Specifically, the background genes in the GENE2FUNC is there for the $\mathrm{N}$ which is supposed to be all the genes we considered to select a set of interested genes n. And we have a tested gene set with $\mathrm{m}$ genes. The number of overlapped genes between $\mathrm{n}$ and $\mathrm{m}$ is $\mathrm{x}$. Therefore, the null hypothesis is finding $\mathrm{x}$ genes given $\mathrm{N}, \mathrm{n}$ and $\mathrm{m}$ is not more than expected. For example, the GWAS catalog gene sets were defined by extracting genes for each trait from the GWAS catalog. Using the GENE2FUNC procedure, we examined whether the mapped genes enriched in some specific diseases or traits in GWAS catalog as well as whether enriched in specific GO, KEGG et al. The significant results were selected if Bonferroni-corrected $P<0.05$.

\section{PPI network analysis}

The PPI network was constructed with the Search Tool for Retrieval of Interacting Genes/Proteins (STRING(Szklarczyk et al., 2019), https://string-db.org/cgi/input.pl/). Given

arst of the proteins as input, STRING can search for their neighbor interactors, the proteins

614 that have direct interactions with the inputted proteins; then STRING can generate the PPI 615 network consisting of all these proteins and all the interactions between them. We first 616 constructed the PPI network with the 47 significant genes as input, the network displayed on 617 the webpage was gathered into two main clusters and then exported as a high-resolution 618 bitmap. Meanwhile, we got the KEGG pathway enrichment results which were used to 619 characterize the biological importance of the clusters.

623 We compared the difference of diversity and microbiota composition between genders. 624 Diversity was calculated for Shannon index based on genus-level relative abundance of 625 microbial taxa. Pairwise comparisons were performed using non-parametric test (Wilcoxon 626 test). The multivariate association with linear models (MaAsLin)(Morgan et al., 2012) 627 package was used to identify the differentially abundant taxa between genders. Only taxa 628 with q values $<0.05$ are identified as significantly enriched in males or females. 
We performed gender-specific GWAS analysis in male and female separately using the same methods as described in the microbiome genome-wide association analysis. Malespecific variants were identified as (i) significantly associated with taxa in male $\left(P_{\text {male }}<5 \times\right.$ $\left.10^{-8}\right)$ and not significant in female $\left(P_{\text {female }}>0.05\right)$, and (ii) had nominal significant gender difference (testing $\mathrm{P}$ value for difference in gender-specific effect size estimated by beta value, $\left.P_{\text {difference }}<0.01\right)$. Female-specific variants were identified as (i) significantly associated with taxa in female $\left(P_{\text {female }}<5 \times 10^{-8}\right)$ and not significant in male $\left(P_{\text {male }}>0.05\right)$, and (ii) had nominal significant gender-difference $\left(P_{\text {difference }}<0.01\right.$, as explained below $)$.

For each variant (SNP/indel/CNV) and for the phenotype (relative abundance of taxa), we computed $\mathrm{P}$ values $\left(P_{\text {difference }}\right)$ testing for difference between the male-specific and femalespecific beta-estimates $b_{\text {male }}$ and $b_{\text {female }}$ using the $T$-statistic

$\left(b_{\text {male }}-b_{\text {female }}\right) / \operatorname{sqrt}\left(\mathrm{se}_{\text {male }}{ }^{2}+\mathrm{se}_{\text {female }}{ }^{2}-2 *\right.$ corr $\left.\left(b_{\text {male }}, b_{\text {female }}\right) * s_{\text {male }} * s_{\text {female }}\right)$

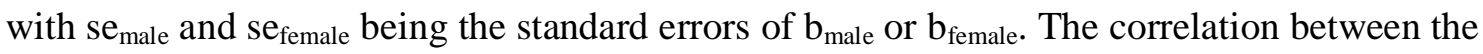
gender-specific beta estimates was computed as the Spearman rank correlation coefficient across all variants for each phenotype.

\section{Gene expression and differential analysis}

We used GEPIA(Tang et al., 2017) (Gene Expression Profiling Interactive Analysis), a webbased tool to deliver fast and customizable functionalities based on the Cancer Genome Atlas (TCGA) and Genotype-Tissue Expression (GTEx) data. We performed the differential expression analysis for genes $N X N$ and $P A R V B$ across colon adenocarcinoma (COAD) and rectum adenocarcinoma (READ) types compared with paired normal samples, respectively. We choose $\log 2($ TPM +1$)$ transformed expression data for plotting. We used ANOVA method for differential analysis. Genes with higher $\left|\log _{2} \mathrm{FC}\right|>0.2$ and $\mathrm{p}$ values $<0.05$ are considered differentially expressed genes.

\section{DATA AND SOFTWARE AVAILABILITY}

The data in this study have been deposited to the CNGB Nucleotide Sequence Archive (CNSA: https://db.cngb.org/cnsa; accession number CNP0000289).

\section{Acknowledgments}

We are very grateful to colleagues at BGI-Shenzhen for sample collection, DNA extraction, library construction, sequencing, and discussions.

\section{Author contributions}

H.J. and T.Z. conceived and organized this study. J.W. initiated the overall health project. X.X., H.Y. and Y.H. performed the sample collection and questionnaire collection. X.M.L., T.Z., X.T., R.G., Y.Z., X.L., Y.Z. and Y.H. generated and processed the whole genome data. S.T., H.Z., J.Z., Q.D., D.W., L.X. and K.K. generated and processed the metagenome data. X.M.L., S.T., and S.B. performed the bioinformatic analyses, X.M.L. and H.J. wrote the manuscript. All authors contributed to data and texts in this manuscript. 


\section{Declaration of interests}

674 The authors declare no competing financial interest.

\section{Reference}

Atarashi, K., Suda, W., Luo, C., Kawaguchi, T., Motoo, I., Narushima, S., Kiguchi, Y., Yasuma, K., 679 Watanabe, E., Tanoue, T., et al. (2017). Ectopic colonization of oral bacteria in the intestine 680 drives TH1 cell induction and inflammation. Science 358, 359-365.

681 Blacher, E., Bashiardes, S., Shapiro, H., Rothschild, D., Mor, U., Dori-Bachash, M., Kleimeyer, C., 682 Moresi, C., Harnik, Y., Zur, M., et al. (2019). Potential roles of gut microbiome and metabolites 683 in modulating ALS in mice. Nature.

684 Blekhman, R., Goodrich, J.K., Huang, K., Sun, Q., Bukowski, R., Bell, J.T., Spector, T.D., Keinan, A., 685 Ley, R.E., Gevers, D., et al. (2015). Host genetic variation impacts microbiome composition 686 across human body sites. Genome biology 16, 191.

687 Bonder, M.J., Kurilshikov, A., Tigchelaar, E.F., Mujagic, Z., Imhann, F., Vila, A.V., Deelen, P., 688 Vatanen, T., Schirmer, M., Smeekens, S.P., et al. (2016). The effect of host genetics on the gut 689 microbiome. Nature genetics 48, 1407-1412.

690 Brand, G., and Millot, J.L. (2001). Sex differences in human olfaction: between evidence and 691 enigma. The Quarterly journal of experimental psychology B, Comparative and physiological 692 psychology 54, 259-270.

693 Bravou, V., Antonacopoulou, A., Papanikolaou, S., Nikou, S., Lilis, I., Giannopoulou, E., and 694 Kalofonos, H.P. (2015). Focal Adhesion Proteins alpha- and beta-Parvin are Overexpressed in 695 Human Colorectal Cancer and Correlate with Tumor Progression. Cancer investigation 33, 696 387-397.

697 Browning, B.L., Zhou, Y., and Browning, S.R. (2018). A One-Penny Imputed Genome from 698 Next-Generation Reference Panels. Am J Hum Genet 103, 338-348.

699 Bychkov, E., Ahmed, M.R., and Gurevich, E.V. (2011). Sex differences in the activity of 700 signalling pathways and expression of G-protein-coupled receptor kinases in the neonatal 701 ventral hippocampal lesion model of schizophrenia. The international journal of 702 neuropsychopharmacology 14, 1-15.

703 Cao, Y., Lin, W., and Li, H. (2019). Large Covariance Estimation for Compositional Data Via 704 Composition-Adjusted Thresholding. Journal of the American Statistical Association 114, 705 759-772.

706 Caspi, R., Altman, T., Billington, R., Dreher, K., Foerster, H., Fulcher, C.A., Holland, T.A., Keseler, 707 I.M., Kothari, A., Kubo, A., et al. (2014). The MetaCyc database of metabolic pathways and 708 enzymes and the BioCyc collection of Pathway/Genome Databases. Nucleic Acids Res 42, 709 D459-471. 
710 Chiang, C., Layer, R.M., Faust, G.G., Lindberg, M.R., Rose, D.B., Garrison, E.P., Marth, G.T.,

711 Quinlan, A.R., and Hall, I.M. (2015). SpeedSeq: ultra-fast personal genome analysis and

712 interpretation. Nat Methods 12, 966-968.

713 Comuzzie, A.G., Cole, S.A., Laston, S.L., Voruganti, V.S., Haack, K., Gibbs, R.A., and Butte, N.F.

714 (2012). Novel genetic loci identified for the pathophysiology of childhood obesity in the

715 Hispanic population. PLoS One 7, e51954.

716 Costea, P.I., Hildebrand, F., Arumugam, M., Backhed, F., Blaser, M.J., Bushman, F.D., de Vos,

717 W.M., Ehrlich, S.D., Fraser, C.M., Hattori, M., et al. (2018). Enterotypes in the landscape of gut

718 microbial community composition. Nature microbiology 3, 8-16.

719 Dhakan, D.B., Maji, A., Sharma, A.K., Saxena, R., Pulikkan, J., Grace, T., Gomez, A., Scaria, J.,

720 Amato, K.R., and Sharma, V.K. (2019). The unique composition of Indian gut microbiome,

721 gene catalogue, and associated fecal metabolome deciphered using multi-omics approaches.

722 GigaScience 8.

723 Ding, T., and Schloss, P.D. (2014). Dynamics and associations of microbial community types

724 across the human body. Nature 509, 357-360.

725 Fang, C., Zhong, H., Lin, Y., Chen, B., Han, M., Ren, H., Lu, H., Luber, J.M., Xia, M., Li, W., et al.

726 (2018). Assessment of the CPAS-based BGISEQ-500 platform for metagenomic sequencing.

727 GigaScience 7, 1-8.

728 Goodrich, J.K., Davenport, E.R., Beaumont, M., Jackson, M.A., Knight, R., Ober, C., Spector, T.D.,

729 Bell, J.T., Clark, A.G., and Ley, R.E. (2016). Genetic Determinants of the Gut Microbiome in UK

730 Twins. Cell host \& microbe 19, 731-743.

731 Gusev, A., Ko, A., Shi, H., Bhatia, G., Chung, W., Penninx, B.W., Jansen, R., de Geus, E.J.,

732 Boomsma, D.I., Wright, F.A., et al. (2016). Integrative approaches for large-scale

733 transcriptome-wide association studies. Nature genetics 48, 245-252.

734 Han, M., Hao, L., Lin, Y., Li, F., Wang, J., Yang, H., Xiao, L., Kristiansen, K., Jia, H., and Li, J. (2018).

735 A novel affordable reagent for room temperature storage and transport of fecal samples for

736 metagenomic analyses. Microbiome 6, 43.

737 Holmes, I., Harris, K., and Quince, C. (2012). Dirichlet multinomial mixtures: generative models

738 for microbial metagenomics. PLoS One 7, e30126.

739 Jeffery, I.B., Claesson, M.J., O'Toole, P.W., and Shanahan, F. (2012). Categorization of the gut

740 microbiota: enterotypes or gradients? Nature reviews Microbiology 10, 591-592.

741 Jie, Z., Chen, C., Hao, L., Li, F., Song, L., Zhang, X., Tian, L., Tong, X., Cai, K., Ju, Y., et al. (2019a).

742 Life history recorded in the vagino-cenical microbiome. bioRxiv, 533588.

743 Jie, Z., Liang, S., Ding, Q., Tang, S., Li, F., Wang, D., Lin, Y., Chen, P., Cai, K., Rao, W., et al.

744 (2019b). A multi-omic cohort as a reference point for promoting a healthy human gut

745 microbiome. bioRxiv, 585893.

746 Jie, Z., Xia, H., Zhong, S.L., Feng, Q., Li, S., Liang, S., Zhong, H., Liu, Z., Gao, Y., Zhao, H., et al.

747 (2017). The gut microbiome in atherosclerotic cardiovascular disease. Nature

748 communications 8,845 . 
749 Kanehisa, M., Goto, S., Sato, Y., Kawashima, M., Furumichi, M., and Tanabe, M. (2014). Data,

750 information, knowledge and principle: back to metabolism in KEGG. Nucleic Acids Res 42,

751 D199-205.

752 Khramtsova, E.A., Davis, L.K., and Stranger, B.E. (2018). The role of sex in the genomics of

753 human complex traits. Nature reviews Genetics.

754 Kneeshaw, S., Keyani, R., Delorme-Hinoux, V., Imrie, L., Loake, G.J., Le Bihan, T., Reichheld, J.P.,

755 and Spoel, S.H. (2017). Nucleoredoxin guards against oxidative stress by protecting

756 antioxidant enzymes. Proc Natl Acad Sci U S A.

757 Knights, D., Ward, T.L., McKinlay, C.E., Miller, H., Gonzalez, A., McDonald, D., and Knight, R.

758 (2014). Rethinking "enterotypes". Cell host \& microbe 16, 433-437.

759 Leslie, R., O'Donnell, C.J., and Johnson, A.D. (2014). GRASP: analysis of genotype-phenotype

760 results from 1390 genome-wide association studies and corresponding open access

761 database. Bioinformatics 30, i185-194.

$762 \mathrm{Li}, \mathrm{H}$. , and Durbin, R. (2009). Fast and accurate short read alignment with Burrows-Wheeler

763 transform. Bioinformatics 25, 1754-1760.

764 Li, H., Handsaker, B., Wysoker, A., Fennell, T., Ruan, J., Homer, N., Marth, G., Abecasis, G.,

765 Durbin, R., and Genome Project Data Processing, S. (2009a). The Sequence Alignment/Map

766 format and SAMtools. Bioinformatics 25, 2078-2079.

767 Li, J., Jia, H., Cai, X., Zhong, H., Feng, Q., Sunagawa, S., Arumugam, M., Kultima, J.R., Prifti, E.,

768 Nielsen, T., et al. (2014). An integrated catalog of reference genes in the human gut

769 microbiome. Nat Biotechnol 32, 834-841.

770 Li, R., Yu, C., Li, Y., Lam, T.W., Yiu, S.M., Kristiansen, K., and Wang, J. (2009b). SOAP2: an

771 improved ultrafast tool for short read alignment. Bioinformatics 25, 1966-1967.

772 Lim, M.Y., You, H.J., Yoon, H.S., Kwon, B., Lee, J.Y., Lee, S., Song, Y.M., Lee, K., Sung, J., and Ko,

773 G. (2017). The effect of heritability and host genetics on the gut microbiota and metabolic

774 syndrome. Gut 66, 1031-1038.

775 Liu, R., Hong, J., Xu, X., Feng, Q., Zhang, D., Gu, Y., Shi, J., Zhao, S., Liu, W., Wang, X., et al.

776 (2017). Gut microbiome and serum metabolome alterations in obesity and after weight-loss

777 intervention. Nat Med 23, 859-868.

778 MacArthur, J., Bowler, E., Cerezo, M., Gil, L., Hall, P., Hastings, E., Junkins, H., McMahon, A.,

779 Milano, A., Morales, J., et al. (2017). The new NHGRI-EBI Catalog of published genome-wide

780 association studies (GWAS Catalog). Nucleic Acids Res 45, D896-D901.

781 MacDonald, J.R., Ziman, R., Yuen, R.K., Feuk, L., and Scherer, S.W. (2014). The Database of

782 Genomic Variants: a curated collection of structural variation in the human genome. Nucleic

783 Acids Res 42, D986-992.

784 McKenna, A., Hanna, M., Banks, E., Sivachenko, A., Cibulskis, K., Kernytsky, A., Garimella, K.,

785 Altshuler, D., Gabriel, S., Daly, M., et al. (2010). The Genome Analysis Toolkit: a MapReduce

786 framework for analyzing next-generation DNA sequencing data. Genome Res 20, 1297-1303. 
787

788

789

790

791

792

793

794

795

796

797

798

799

800

801

802

803

804

805

806

807

808

809

810

811

812

813

814

815

816

817

818

819

820

821

822

823

824

825

Morgan, X.C., Tickle, T.L., Sokol, H., Gevers, D., Devaney, K.L., Ward, D.V., Reyes, J.A., Shah, S.A., LeLeiko, N., Snapper, S.B., et al. (2012). Dysfunction of the intestinal microbiome in inflammatory bowel disease and treatment. Genome biology 13, R79.

Oliver, P.L., Finelli, M.J., Edwards, B., Bitoun, E., Butts, D.L., Becker, E.B., Cheeseman, M.T., Davies, B., and Davies, K.E. (2011). Oxr1 is essential for protection against oxidative stressinduced neurodegeneration. PLoS genetics 7, e1002338.

Onderdonk, A.B., Delaney, M.L., and Fichorova, R.N. (2016). The Human Microbiome during Bacterial Vaginosis. Clin Microbiol Rev 29, 223-238.

Org, E., Parks, B.W., Joo, J.W., Emert, B., Schwartzman, W., Kang, E.Y., Mehrabian, M., Pan, C., Knight, R., Gunsalus, R., et al. (2015). Genetic and environmental control of host-gut microbiota interactions. Genome Res 25, 1558-1569.

Perry, J.R., McCarthy, M.I., Hattersley, A.T., Zeggini, E., Wellcome Trust Case Control, C., Weedon, M.N., and Frayling, T.M. (2009). Interrogating type 2 diabetes genome-wide association data using a biological pathway-based approach. Diabetes 58, 1463-1467.

Pruitt, K.D., Tatusova, T., Brown, G.R., and Maglott, D.R. (2012). NCBI Reference Sequences (RefSeq): current status, new features and genome annotation policy. Nucleic Acids Res 40, D130-135.

Purcell, S., Neale, B., Todd-Brown, K., Thomas, L., Ferreira, M.A., Bender, D., Maller, J., Sklar, P., de Bakker, P.I., Daly, M.J., et al. (2007). PLINK: a tool set for whole-genome association and population-based linkage analyses. Am J Hum Genet 81, 559-575.

Qin, J., Li, Y., Cai, Z., Li, S., Zhu, J., Zhang, F., Liang, S., Zhang, W., Guan, Y., Shen, D., et al. (2012). A metagenome-wide association study of gut microbiota in type 2 diabetes. Nature 490, 55-60.

Rothschild, D., Weissbrod, O., Barkan, E., Kurilshikov, A., Korem, T., Zeevi, D., Costea, P.I., Godneva, A., Kalka, I.N., Bar, N., et al. (2018). Environment dominates over host genetics in shaping human gut microbiota. Nature 555, 210-215.

Szklarczyk, D., Gable, A.L., Lyon, D., Junge, A., Wyder, S., Huerta-Cepas, J., Simonovic, M., Doncheva, N.T., Morris, J.H., Bork, P., et al. (2019). STRING v11: protein-protein association networks with increased coverage, supporting functional discovery in genome-wide experimental datasets. Nucleic Acids Res 47, D607-D613.

Tang, Z., Li, C., Kang, B., Gao, G., Li, C., and Zhang, Z. (2017). GEPIA: a web server for cancer and normal gene expression profiling and interactive analyses. Nucleic Acids Res 45, W98W102.

Turpin, W., Espin-Garcia, O., Xu, W., Silverberg, M.S., Kevans, D., Smith, M.I., Guttman, D.S., Griffiths, A., Panaccione, R., Otley, A., et al. (2016). Association of host genome with intestinal microbial composition in a large healthy cohort. Nature genetics 48, 1413-1417.

Vandeputte, D., Kathagen, G., D'Hoe, K., Vieira-Silva, S., Valles-Colomer, M., Sabino, J., Wang, J., Tito, R.Y., De Commer, L., Darzi, Y., et al. (2017). Quantitative microbiome profiling links gut community variation to microbial load. Nature 551, 507-511. 
826 Vangay, P., Johnson, A.J., Ward, T.L., Al-Ghalith, G.A., Shields-Cutler, R.R., Hillmann, B.M., 827 Lucas, S.K., Beura, L.K., Thompson, E.A., Till, L.M., et al. (2018). US Immigration Westernizes

828 the Human Gut Microbiome. Cell 175, 962-972 e910.

829 Vieira-Silva, S., Falony, G., Darzi, Y., Lima-Mendez, G., Garcia Yunta, R., Okuda, S., Vandeputte, 830 D., Valles-Colomer, M., Hildebrand, F., Chaffron, S., et al. (2016). Species-function 831 relationships shape ecological properties of the human gut microbiome. Nature 832 microbiology 1, 16088.

833 Wang, J., and Jia, H. (2016). Metagenome-wide association studies: fine-mining the 834 microbiome. Nature reviews Microbiology 14, 508-522.

835 Wang, J., Thingholm, L.B., Skieceviciene, J., Rausch, P., Kummen, M., Hov, J.R., Degenhardt, F., 836 Heinsen, F.A., Ruhlemann, M.C., Szymczak, S., et al. (2016). Genome-wide association analysis 837 identifies variation in vitamin $D$ receptor and other host factors influencing the gut 838 microbiota. Nature genetics 48, 1396-1406.

839 Watanabe, K., Taskesen, E., van Bochoven, A., and Posthuma, D. (2017). Functional mapping 840 and annotation of genetic associations with FUMA. Nature communications 8, 1826.

841 Wojcik, G.L., Graff, M., Nishimura, K.K., Tao, R., Haessler, J., Gignoux, C.R., Highland, H.M., 842 Patel, Y.M., Sorokin, E.P., Avery, C.L., et al. (2019). Genetic analyses of diverse populations 843 improves discovery for complex traits. Nature 570, 514-518.

844 Wu, M.C., Lee, S., Cai, T., Li, Y., Boehnke, M., and Lin, X. (2011). Rare-variant association 845 testing for sequencing data with the sequence kernel association test. Am J Hum Genet 89, $846 \quad 82-93$.

847 Xiao, L., Estelle, J., Kiilerich, P., Ramayo-Caldas, Y., Xia, Z., Feng, Q., Liang, S., Pedersen, A.O., 848 Kjeldsen, N.J., Liu, C., et al. (2016). A reference gene catalogue of the pig gut microbiome. 849 Nature microbiology, 16161.

850 Xiao, L., Feng, Q., Liang, S., Sonne, S.B., Xia, Z., Qiu, X., Li, X., Long, H., Zhang, J., Zhang, D., et 851 al. (2015). A catalog of the mouse gut metagenome. Nat Biotechnol 33, 1103-1108.

852 Xie, H., Guo, R., Zhong, H., Feng, Q., Lan, Z., Qin, B., Ward, K.J., Jackson, M.A., Xia, Y., Chen, X., 853 et al. (2016). Shotgun Metagenomics of 250 Adult Twins Reveals Genetic and Environmental 854 Impacts on the Gut Microbiome. Cell systems 3, 572-584 e573.

855 Xing Hua, L.S., Guoqin Yu, James J. Goedert, Christian C. Abnet, Maria Teresa Landi, Jianxin 856 Shi (2015). MicrobiomeGWAS: a tool for identifying host genetic variants associated with 857 microbiome composition. bioRxiv.

858 Yang, J., Bakshi, A., Zhu, Z., Hemani, G., Vinkhuyzen, A.A., Lee, S.H., Robinson, M.R., Perry, J.R., 859 Nolte, I.M., van Vliet-Ostaptchouk, J.V., et al. (2015). Genetic variance estimation with 860 imputed variants finds negligible missing heritability for human height and body mass index. 861 Nature genetics 47, 1114-1120.

862 Yang, J., Lee, S.H., Goddard, M.E., and Visscher, P.M. (2011). GCTA: a tool for genome-wide 863 complex trait analysis. Am J Hum Genet 88, 76-82. 
864 Zeng, Y., Nie, C., Min, J., Chen, H., Liu, X., Ye, R., Chen, Z., Bai, C., Xie, E., Yin, Z., et al. (2018).

865 Sex Differences in Genetic Associations With Longevity. JAMA network open 1.

866 Zhang, X., Zhang, D., Jia, H., Feng, Q., Wang, D., Liang, D., Wu, X., Li, J., Tang, L., Li, Y., et al. 867 (2015). The oral and gut microbiomes are perturbed in rheumatoid arthritis and partly

868 normalized after treatment. Nat Med 21, 895-905.

869 Zou, H., Wang, D., Ren, H., Chen, P., Fang, C., Shi, Z., Zhang, P., Wang, J., Yang, H., Cai, K., et al. 870 (2019). Calorie restriction intervention induces enterotype-associated BMI loss in nonobese 871 individuals. bioRxiv, 514596.

872 


\section{Figure captions}

874 Figure 1. Identifying host genetic variants associated with microbiome enterotypes and 875 principal coordinates (PCoAs, computed using Bray-Curtis dissimilarity). (A) The 876 enterotype plot of 618 individuals in discovery cohort. Two clusters were shown with red 877 dots representing Bacteroides-dominant enterotype (440 individuals) and blue dots 878 representing Prevotella-dominant enterotype (178 individuals). The first 2 principal 879 components (PCoA1 and PCoA2) are shown, with the amount of variation explained are 880 reported for each axe. (B) The enterotype plot of 663 individuals in replication cohort. Two 881 clusters were shown with red dots representing Bacteroides-dominant enterotype (473 individuals) and blue dots representing Prevotella-dominant enterotype (190 individuals). (C)The minor allele G of SNP rs13045408 at BTBD3-LINC01722 were positively correlated with Bacteroides abundance and negatively correlated with Prevotella abundance in discovery cohort. However, SNP rs1453213 at OXR1 had opposite effects to enterotypes compared with rs13045408 in (B). (D) rs13045408 and rs1453213 associated with 'Bacteroides-Prevotella' enterotype in replication cohort $\left(P_{P-B}=0.061\right.$ and 0.024 , respectively). (E) Manhattan plots of the host genetic variants associated with microbiome $\beta$-diversity (computed as Bray-Curtis dissimilarity matrix). The red line represents a genome-wide significant $P$ value $\left(5 \times 10^{-8}\right)$ and blue line represents suggestive $\mathrm{P}$ value $\left(10^{-5}\right)$. Five top loci were marked with gene name. (F) The replicated $\mathrm{P}$ value in this study for the 380 SNPs previously reported to be significantly associated with the microbiome. Eleven SNPs are successfully replicated at P $<1.3 \times 10^{-4}=0.05 / 380$ (blue line), nine of which were most associated with Bacteroides stercoris.

Figure 2. Heritability of gut microbial taxa and its functions. (A) The heritability (h2 calculated using GREML-LDMS model in GCTA tool using host whole genome) was plotted as a bar for each genus. Two significantly heritable genura were focus on Prevotella and Bifidobacterium. More detailed results for heritability of taxa are available in Table S2A. (B) Heritability of gut microbial modules (GMMs), which were colored by different metabolic types, including amino acid degradation (red), carbohydrate degradation (green), organic Acid metabolism (blue) and others (purple). Detailed results for heritability of GMMs are available in Table S2C.

Figure 3. M-GWAS results for the gut microbiome and functional analysis. (A) Manhattan plot showing the associations between common variants and taxa, only suggestive associations with $P<10^{-5}$ were showed. The gray line represents a genome-wide significant $\mathrm{P}$ value $5 \times 10^{-8}$. The red lines showed the 37 loci reaching the genome-wide significance. Two high heritable taxa, Actinobacteria linked to rs13420238 at LOC150935 and Prevotellaceae linked to rs1453123 at OXR1, were marked. (B) rs1453123 at OXR1 associated with Prevotellaceae both in discovery $\left(P=1.58 \times 10^{-8}\right)$ and replication $(\mathrm{P}=0.006)$ cohort. (C) rs13420238 at LOC150935 associated with Bifidobacterium longum (Actinobacteria) both in discovery $\left(P=4.09 \times 10^{-9}\right)$ and replication $(P=0.007)$ cohort. $(D)$ rs760646544 near $P L X D C 2$ associated with Roseburia intestinalis both in discovery $\left(P=3.81 \times 10^{-8}\right)$ and replication $(P=0.043)$ cohort. (E) Enriched traits or diseases identified in GWAS catalog using 109 mapped genes from 37 significant loci. (F) Enriched KEGG or GO pathways identified for 109 mapped genes from 37 significant loci. 
919 Figure 4. Gender-specific associations and functional analysis. (A) Manhattan plot 920 showing the male-specific and female-specific associations for gut microbiome. The red line 921 represents the genome-wide $\mathrm{P}$ threshold $\left(5 \times 10^{-8}\right)$. (B) Enriched traits or diseases identified 922 using male-specific associations mapped in GWAS catalog. (C) Enriched KEGG, 923 REACTOME or GO pathways identified for male-specific associations using FUMA tool. (D) 924 Enriched traits or diseases identified using female-specific associations mapped in GWAS 925 catalog. (E) Enriched KEGG, REACTOME or GO pathways identified for female-specific 926 associations using FUMA tool.

927

Figure 5. M-GWAS helps understand biomarkers from MWAS. (A) Venn diagram showing the bacteria that identified associated with genetic variants and were reported in multiple MWAS studies, including type 2 diabetes (T2D), rheumatoid arthritis (RA), atherosclerotic cardiovascular disease (ACVD), colorectal cancer (CRC) and obesity. Bacteria in overlapping areas indicates it was reported in two or more MWAS studies. The bacteria marked in red represents enrichment in cases, and green represents enrichment in controls. (B) A copy loss of 642bp (marked by green circle) in NXN gene was detected in Chinese individuals. (C) Box plots of the $N X N$ gene expression in COAD and READ cases compared with normal tissue samples using GEPIA tool. COAD, colon adenocarcinoma; READ, rectum adenocarcinoma. (D) Region plot of the most significant loci for PARVB gene. Each point represents a SNP or indel and is colored with the $\mathrm{r}^{2}$ value as calculated in this cohort. The lead SNP rs35928604 $\left(\mathrm{P}=1.55 \times 10^{-8}\right)$ is highlighted with red. (E) As in $(\mathbf{C})$, box

940 plots of the $P A R V B$ gene expression in COAD and READ cases compared with normal tissue 941 samples. 
A discovery cohort

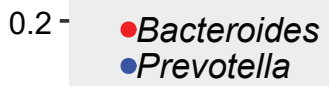

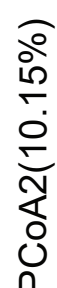

$-0.2-$

$-0.3-$

$$
\begin{array}{ccc}
-0.25 & 0.00 & 0.25
\end{array}
$$

C discovery cohort

\section{Bacteroides Prevotella}

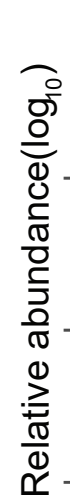

$-4-P_{P-B}=2.1 \times 10^{-6}$

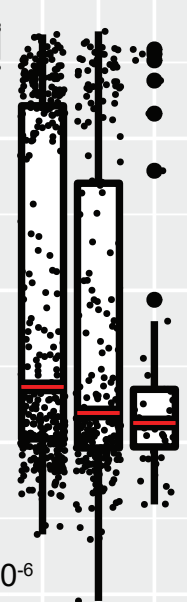

C'C CG G'G C'C C'G G'G rs13045408
Bacteroides Prevotella

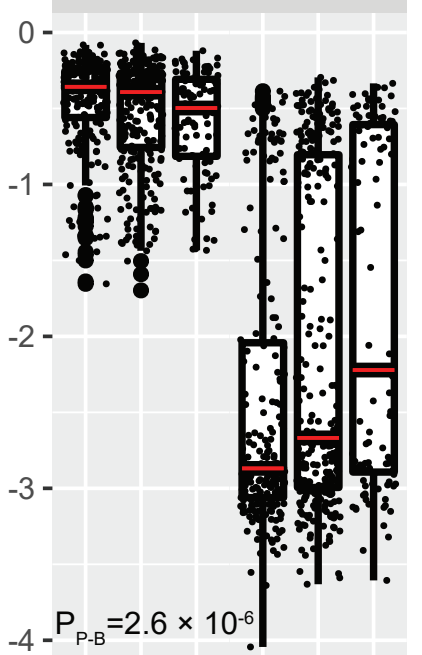

C'С С'т 'ंT 'С С'т TंT rs1453213

$\mathrm{E}$

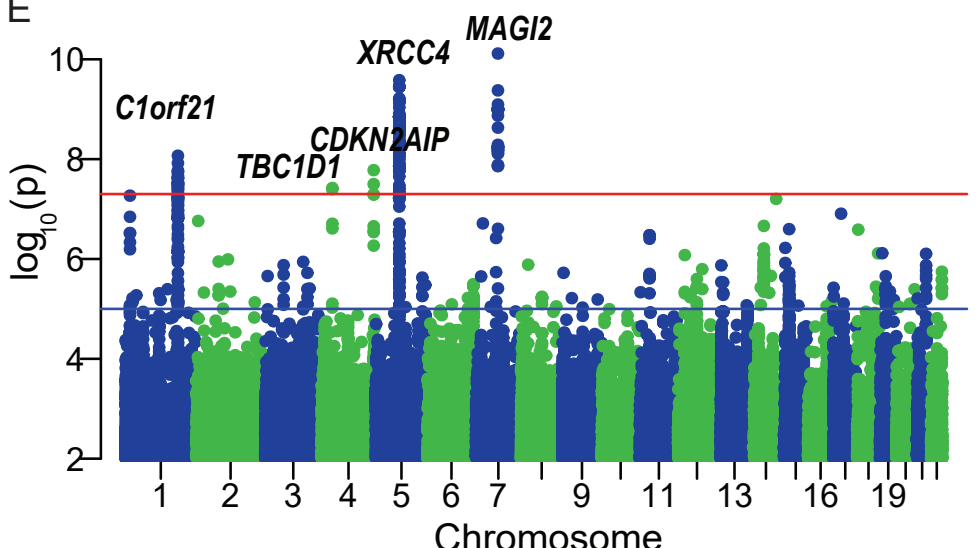

\section{2 -

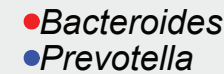

$-0.2-$
0.00

PCoA1(70.43\%)

replication cohort

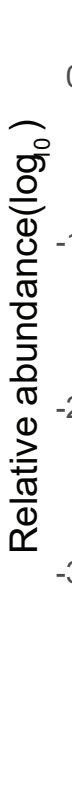

Bacteroides 0 -

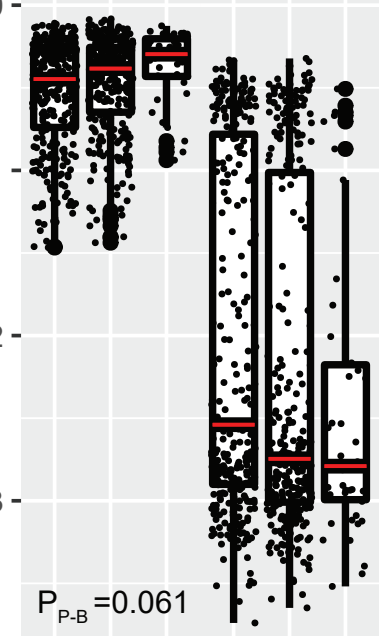

C'C ĆG 'GG C'C C'G G'G rs13045408
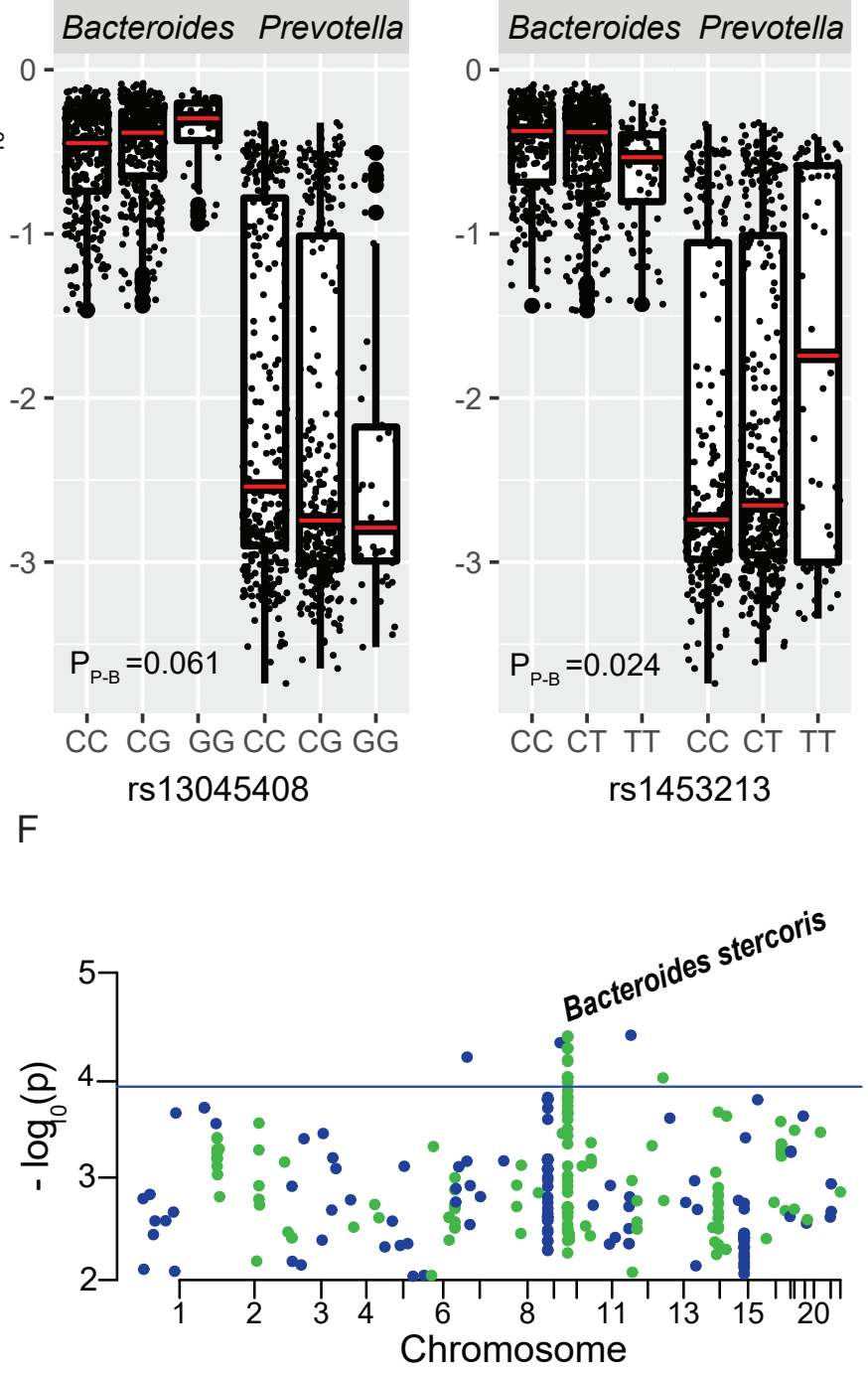

C'C C'T T'T C'C C'T T'T rs1453213 
Actinobacteria Bacteroidetes Firmicutes

Fusobacteria

Lentisphaerae

Proteobacteria

Spirochaetes

Synergistetes

Verrucomicrobia
B

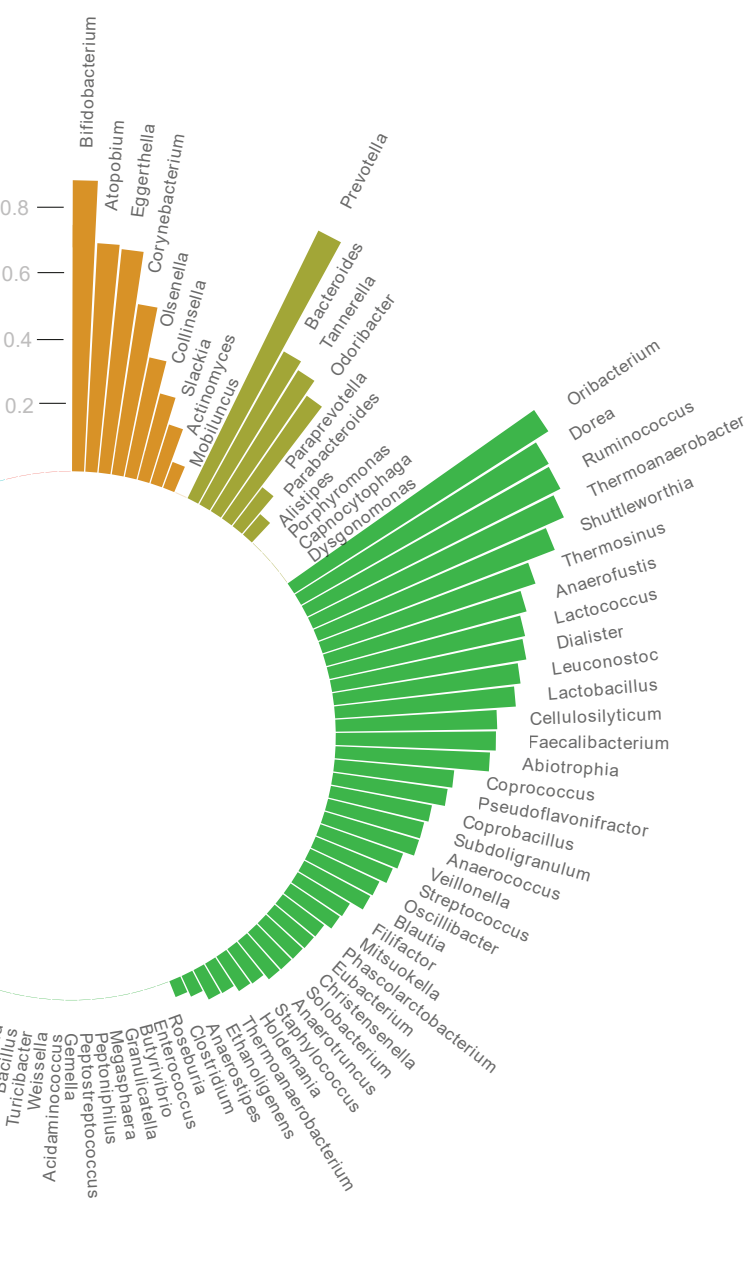

amino acid degradation

carbohydrate degradation

organic acid metabolism

others
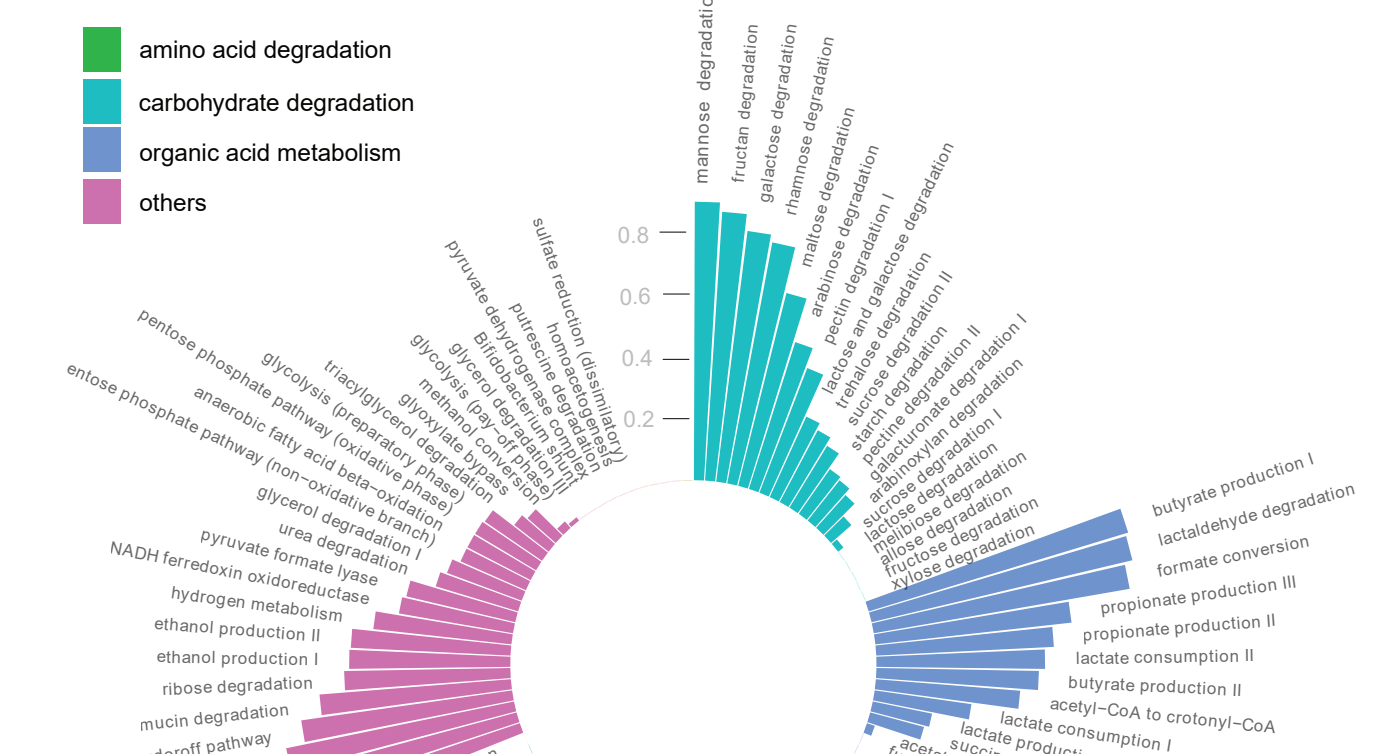

Entner-Doudoroff pathway

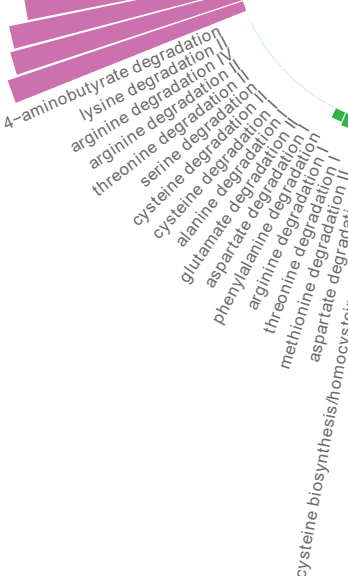

itrate reduction (dis simitiatory)

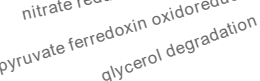
lactate late consuma to crotonyl-CoA

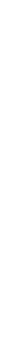




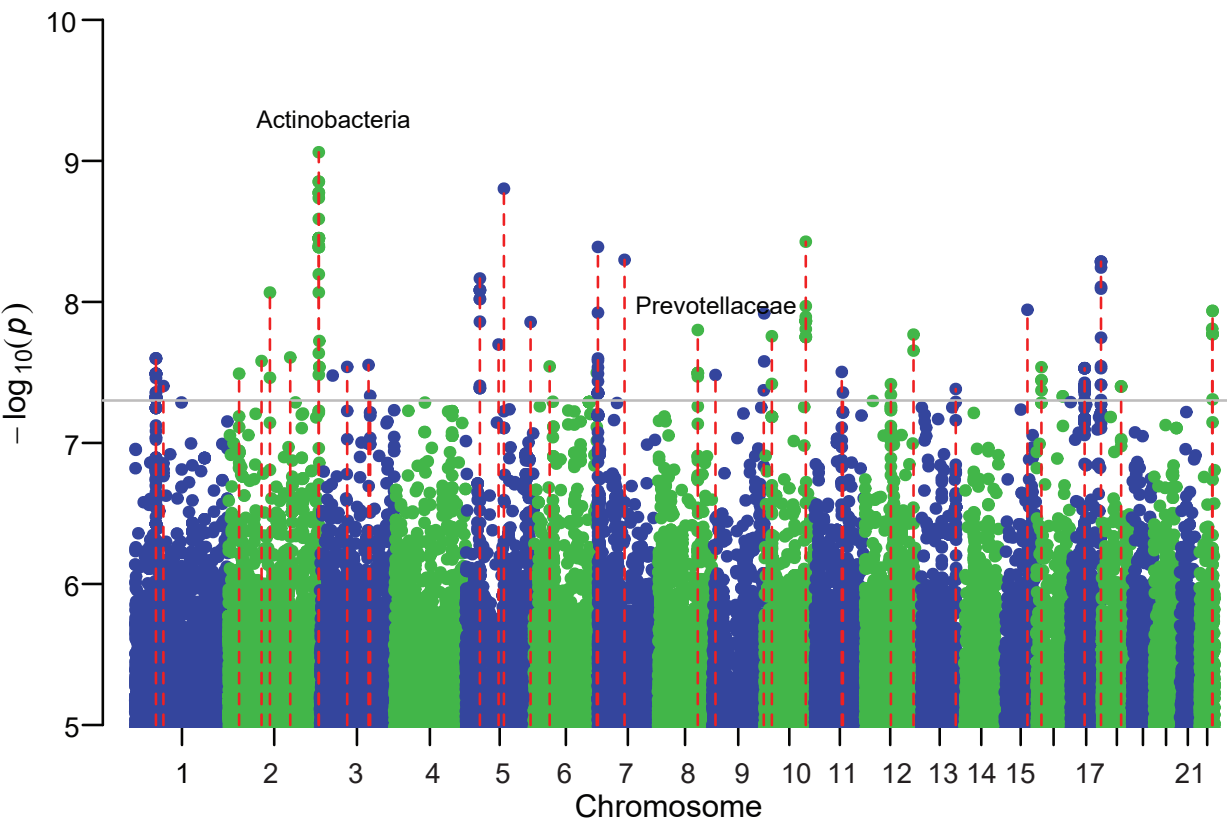

E

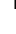

Ovarian cancerBlood osmolality (transformed sodium) Pediatric non-alcoholic fatty liver disease activity scoreWaist Circumference - Triglycerides (WC-TG) -
Metabolite levels (HVA) Metabolite levels (HVA)Acute insulin response-
Immune response to measles-mumps-rubella vaccineMetabolite levels (HVA/MHPG ratio)Palmitoleic acid (16:1n-7) levels-
Periodontitis (CDC/AAP)Periodontitis (CDC/AAP) -
Loneliness

Serum uric acid levels in response to allopurinol in goutInflammatory skin diseaseHematological and biochemical traits Fasting blood glucose (BMI interaction)-
Post bronchodilator FEV1 in COPDImmune response to smallpox vaccine (IL-6) Fasting blood glucose Severe influenza $A(\mathrm{H} 1 \mathrm{~N} 1)$ infectionHemoglobin concentration Systemic lupus erythematosus Type 2 diabetes
Total cholesterol levels Pulse pressurePursuit maintenance gainHDL cholesterol levels Alzheimer's disease (cognitive decline)-
C

D

Prevotellaceae

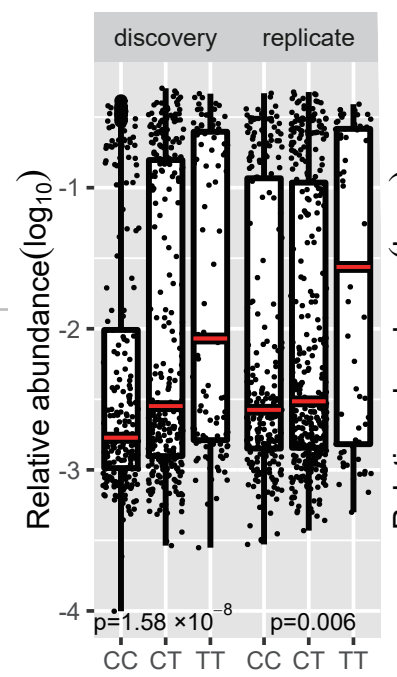

rs 1453213
Bifidobacterium longum

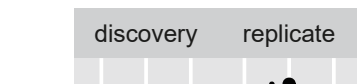

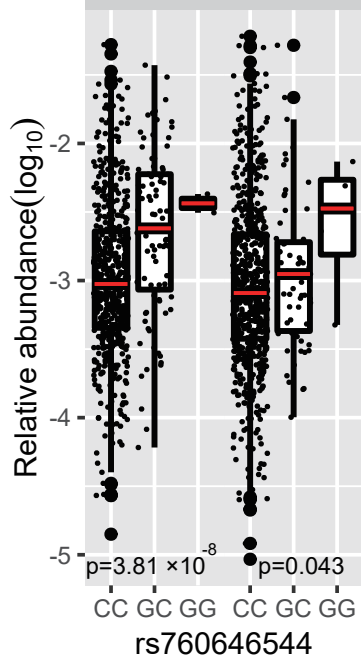

Proportion of overlapping Enrichment P-value genes in gene sets

GO PROTEIN DEMANNOSYLATION GO DIACYLGLYCEROL METABOLIC PROCESS

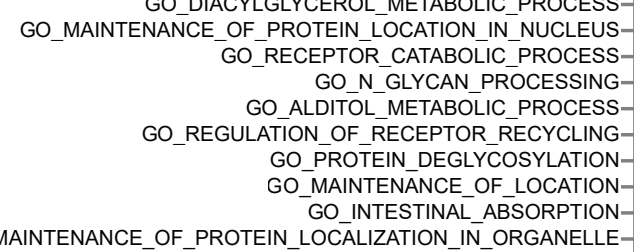
GO_MAINTENANCE_OF_PROTEIN_LOCATION_IN_NUCLEUS
GO_RECEPTOR_CATABOLIC_PROCESS-
GO_N_GLYCAN_PROCESSING-
GO_ALDITOL_METABOLIC_PROCESS
GO_REGULATION_OF_RECEPTOR_RECYCLING-
GO_PROTEIN_DEGLYCOSYLATION
GO_MAINTENANCE_OF_LOCATION
GO_INTESTINAL_ABSORPTION-
GIINTENANCE_OF_PROTEIN_LOCALIZATION_IN_ORGANELLEGO_MAINTENANCE_OF_PROTEIN_LOCATION_IN_NUCLEUS
GO_RECEPTOR_CATABOLIC_PROCESS-
GO_N_GLYCAN_PROCESSING-
GO_ALDITOL_METABOLIC_PROCESS
GO_REGULATION_OF_RECEPTOR_RECYCLING-
GO_PROTEIN_DEGLYCOSYLATION
GO_MAINTENANCE_OF_LOCATION
GO_INTESTINAL_ABSORPTION-
GIINTENANCE_OF_PROTEIN_LOCALIZATION_IN_ORGANELLEGO_MAINTENANCE_OF_PROTEIN_LOCATION_IN_NUCLEUS
GO_RECEPTOR_CATABOLIC_PROCESS-
GO_N_GLYCAN_PROCESSING-
GO_ALDITOL_METABOLIC_PROCESS
GO_REGULATION_OF_RECEPTOR_RECYCLING-
GO_PROTEIN_DEGLYCOSYLATION
GO_MAINTENANCE_OF_LOCATION
GO_INTESTINAL_ABSORPTION-
GIINTENANCE_OF_PROTEIN_LOCALIZATION_IN_ORGANELLEGO_MAINTENANCE_OF_PROTEIN_LOCATION_IN_NUCLEUS
GO_RECEPTOR_CATABOLIC_PROCESS-
GO_N_GLYCAN_PROCESSING-
GO_ALDITOL_METABOLIC_PROCESS
GO_REGULATION_OF_RECEPTOR_RECYCLING-
GO_PROTEIN_DEGLYCOSYLATION
GO_MAINTENANCE_OF_LOCATION
GO_INTESTINAL_ABSORPTION-
GIINTENANCE_OF_PROTEIN_LOCALIZATION_IN_ORGANELLEGO_MAINTENANCE_OF_PROTEIN_LOCATION_IN_NUCLEUS
GO_RECEPTOR_CATABOLIC_PROCESS-
GO_N_GLYCAN_PROCESSING-
GO_ALDITOL_METABOLIC_PROCESS
GO_REGULATION_OF_RECEPTOR_RECYCLING-
GO_PROTEIN_DEGLYCOSYLATION
GO_MAINTENANCE_OF_LOCATION
GO_INTESTINAL_ABSORPTION-
GIINTENANCE_OF_PROTEIN_LOCALIZATION_IN_ORGANELLEGO_MAINTENANCE_OF_PROTEIN_LOCATION_IN_NUCLEUS
GO_RECEPTOR_CATABOLIC_PROCESS-
GO_N_GLYCAN_PROCESSING-
GO_ALDITOL_METABOLIC_PROCESS
GO_REGULATION_OF_RECEPTOR_RECYCLING-
GO_PROTEIN_DEGLYCOSYLATION
GO_MAINTENANCE_OF_LOCATION
GO_INTESTINAL_ABSORPTION-
GIINTENANCE_OF_PROTEIN_LOCALIZATION_IN_ORGANELLEGO_MAINTENANCE_OF_PROTEIN_LOCATION_IN_NUCLEUS
GO_RECEPTOR_CATABOLIC_PROCESS-
GO_N_GLYCAN_PROCESSING-
GO_ALDITOL_METABOLIC_PROCESS
GO_REGULATION_OF_RECEPTOR_RECYCLING-
GO_PROTEIN_DEGLYCOSYLATION
GO_MAINTENANCE_OF_LOCATION
GO_INTESTINAL_ABSORPTION-
GIINTENANCE_OF_PROTEIN_LOCALIZATION_IN_ORGANELLEGO_NEUTRAL_LIPID_BIOSYNTHETIC_PROCESSGO_ALCOHOL_METABOLIC_PROCESSGO_POSITIVE_REGULATION_PO_LEIPID_METABOLIC_PROCESSGO_LIPID_LOCALIZATION

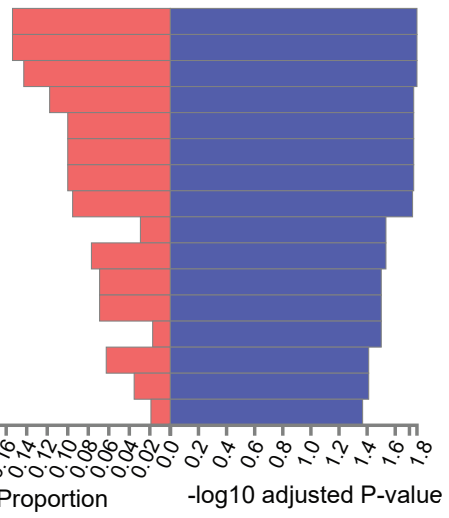


A
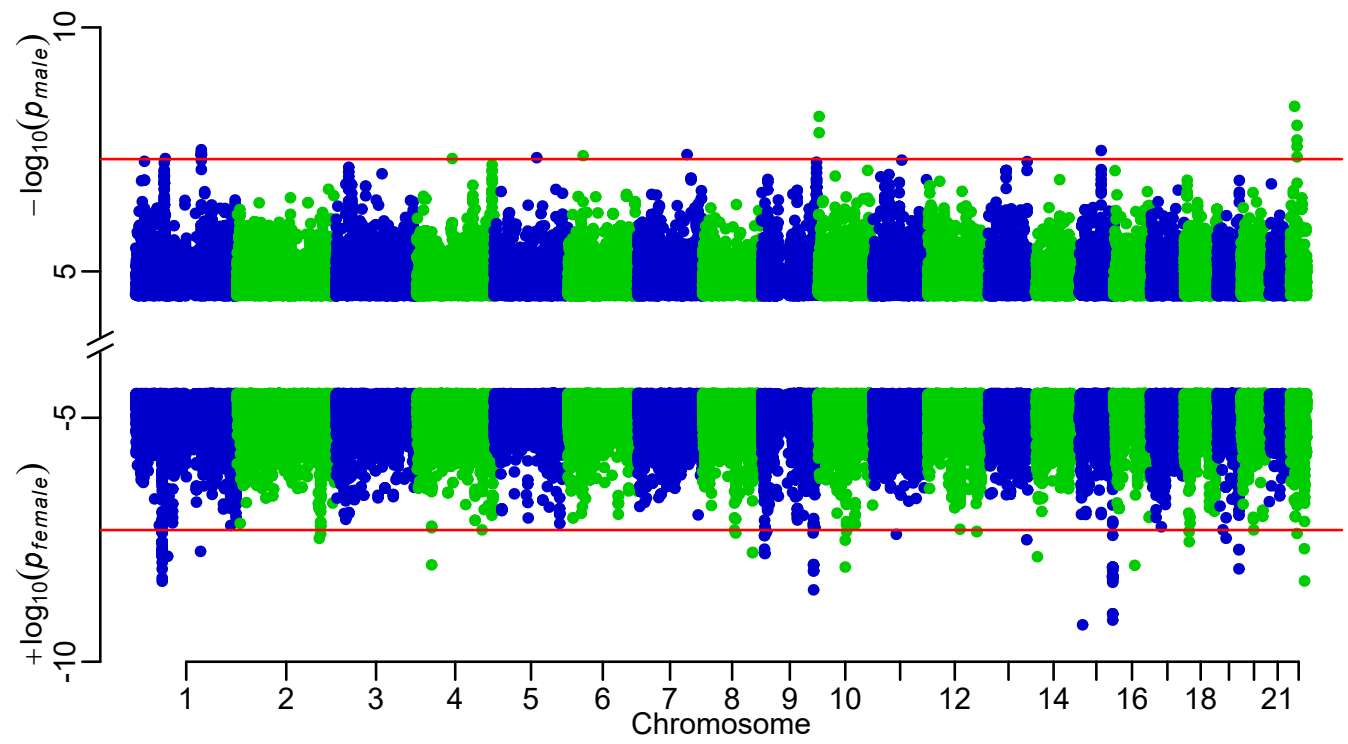

B

Response to cyclophosphamide

in systemic lupus erythematosus with lupus nephritis Interferon alpha levels in systemic lupus erythematosus

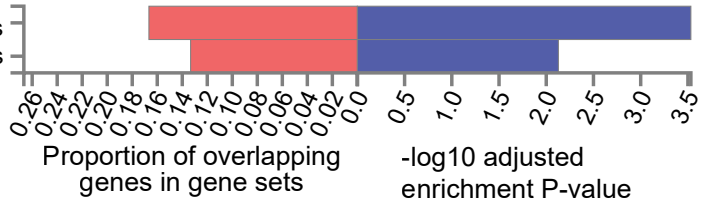

C

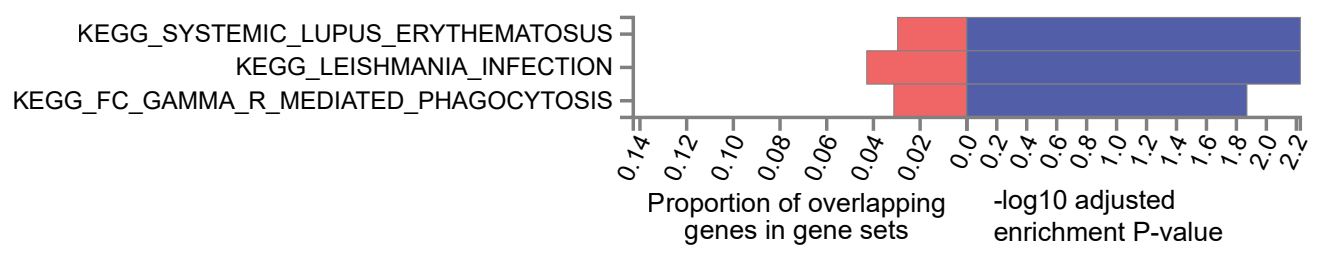

D

Cerebral amyloid deposition in APOEe4 non-carriers (PET imaging) Metabolite levels (HVA) Venous thromboembolism adjusted for sickle cell variant rs77121243-T C-reactive protein levels or triglyceride levels (pleiotropy)

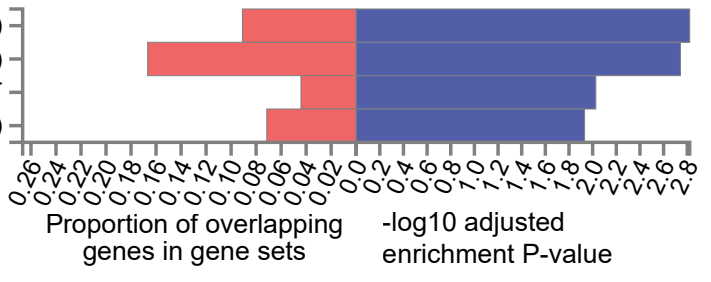

E REACTOME_OLFACTORY_SIGNALING_PATHWAY
KEGG_OLFACTORY_TRANSDUCTION
REACTOME_SIGNALING_BY_GPCR -
REACTOME_GPCR_DOWNSTREAM_SIGNALING

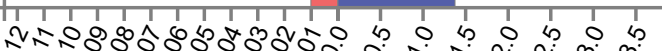
0.000 .000 .000 .000

Proportion of overlapping genes in gene sets

- $\log 10$ adjusted enrichment $P$-value 
